\title{
Co-localization of different Neurotransmitter Transporters on the same Synaptic Vesicle is Bona-fide yet Sparse
}

Neha Upmanyu ${ }^{1,2}$, Jialin Jin ${ }^{3}$, Marcelo Ganzella ${ }^{2}$, Leon Bösche ${ }^{1,2}$, Viveka Nand Malviya ${ }^{2}$, Evi Zhuleku $^{2}$, Antonio Politi ${ }^{4}$, Momchil Ninov ${ }^{5,6}$, Ivan Silbern ${ }^{5,6}$, Henning Urlaub ${ }^{5,6}$, Dietmar Riedel ${ }^{7}$, Julia Preobraschenski ${ }^{2,8}$, Ira Milosevic ${ }^{9,10}$, Reinhard Jahn², Sivakumar Sambandan ${ }^{1,2,11, *}$

\section{Affiliations:}

${ }^{1}$ Synaptic Metal Ion Dynamics and Signaling, Max Planck Institute for Biophysical Chemistry, Göttingen, Germany.

${ }^{2}$ Department of Neurobiology, Max Planck Institute for Biophysical Chemistry, Göttingen, Germany.

${ }^{3}$ European Neurosciences Institute - A Joint Initiative of the University Medical Center Göttingen and the Max Planck Society, Göttingen, Germany

${ }^{4}$ Live-cell Imaging Facility, Max Planck Institute for Biophysical Chemistry, Göttingen, Germany. .

${ }^{5}$ Bioanalytical Mass Spectrometry, Max Planck Institute for Biophysical Chemistry, Göttingen, Germany.

${ }^{6}$ Institute of Clinical Chemistry, University Medical Center Goettingen, Goettingen, Germany

${ }^{7}$ Department of Structural Dynamics, Max Planck Institute for Biophysical Chemistry, Göttingen, Germany

${ }^{8}$ Institute for Auditory Neuroscience, University Medical Center, Göttingen, Germany.

${ }^{9}$ Wellcome Centre for Human Genetics, Nuffield Department of Medicine, NIHR Oxford Biomedical Research Centre, University of Oxford, Oxford, UK

${ }^{10}$ Multidisciplinary Institute of Ageing, MIA-Portugal, University of Coimbra, Coimbra, Portugal

${ }^{11}$ Department of NanoBiophotonics, Max Planck Institute for Biophysical Chemistry, Göttingen, Germany

*Corresponding author. Email: siva.sambandan@mpibpc.mpg.de 


\section{SUMMARY:}

Vesicular transporters (VTs) define the type of neurotransmitter that synaptic vesicles (SVs) store and release. While certain neurons in mammalian brain release multiple transmitters, the prevalence, physiology of such pluralism and if the release occurs from same or distinct vesicle pools is not clear. Using quantitative imaging and biochemical approaches, we show that only a small population of neuronal SVs contain different VTs to accomplish corelease. Surprisingly, a highly diverse SV population (27 types) exist that express dual transporters suggesting corelease of diverse combinations of dual neurotransmitters, which includes the vesicle type that contains glutamate and zinc accounting for $\sim 34 \%$ of all SVs. Importantly, we demonstrate that transporter colocalization influences vesicular glutamate uptake leading to enhanced synaptic quantal size. Thus, localization of diverse transporters on single vesicles is bona-fide and the mechanism may underlie regulation of transmitter content, type and release in space and time.

KEYWORDS: Synaptic vesicle, vesicular transporters, zinc, glutamate, quantal size, DyMIN STED, imaging, Zinc transporter 3, VGLUT1 


\section{INTRODUCTION}

Synaptic vesicles (SVs) are the trafficking organelles responsible for storage and release of various neurotransmitters at the synapse with type and content of transmitters determined by the presence of specific vesicular transporters (VTs) in the SV membrane. Although a given neuron is thought to release only one type of neurotransmitter (referred as Dale's principle), it is well established that certain neurons in the vertebrate central nervous system (CNS) release more than one classical (nonpeptide) neurotransmitter upon activation, referred as 'cotransmission' (Hnasko and Edwards, 2012; Tritsch et al., 2016; Trudeau and El Mestikawy, 2018; Vaaga et al., 2014). Evidence has been mounting, especially in the last decade, that specific neurons release multiple transmitters from the same synapse (Ren et al., 2011; Shabel et al., 2014; Varga et al., 2009). While the prevalence and physiological role of such pluralism is not clear, it has also been difficult to ascertain if the release of multiple neurotransmitters occur from the same pool of vesicles (referred as 'corelease') or segregated pool of SVs that fuse differentially. This knowledge is critical for the understanding function of individual synapse and neuronal circuits.

Corelease of distinct neurotransmitters from same SVs requires that the respective vesicle expresses the corresponding VTs but the available information is not sufficient to confirm the same. For both, corelease and cotransmission, the VTs must be co-expressed in the same neurons. A recent large scale single-cell transcriptomics study (involving $\sim 70,000$ neurons) of the central nervous system reveals that roughly $5 \%$ of neurons co-express multiple VTs indicating the capacity for cotransmission or corelease (Zeisel et al., 2018). An analysis of the data reveal highly diverse neurons expressing up to four different transporters, reflecting an unappreciated complexity of synaptic signaling (Figure S1). Considering the limitations in transcript detection in single-cell studies, one can easily conclude that the actual population of neurons expressing multiple VTs would be much higher, hinting widespread cotransmission or corelease in the brain.

Further discoveries, using optogenetics, in vivo electrophysiology and anatomical approaches, have convincingly established the identity of an array of dual transmitter neurons and their postsynaptic partners in specific brain regions (Ren et al., 2011; Shabel et al., 2014; Takacs et al., 2018; Varga et al., 2009). The two major fast neurotransmitters, glutamate and GABA, that exert excitatory and inhibitory postsynaptic responses, respectively, have been shown to be released together with another transmitter that is modulatory in nature. For example, glutamate is released together with dopamine in striatum and ventral tegmental area, VTA (Chuhma et al., 2004, Stuber et al., 2010, (Hnasko et al., 2010)), or with serotonin in the hippocampal raphe nuclei projections (Sengupta et al., 2017; Varga et al., 2009), or with acetylcholine in striatal interneurons and medial habenula projections (Frahm et al., 2015; Gras et al., 2008; Higley et al., 2011; Ren et al., 2011). Similarly, GABA is cotransmitted with acetylcholine in basal forebrain projections (Saunders et al., 2015; Takacks et al., 2018), or with dopamine in the olfactory bulb (Liu et al., 2013) and striatum (Tritsch et al., 2012). Interestingly, cotransmission of glutamate and GABA have also been observed in some neurons (Root et al., 2018; Shabel et al., 2014) including evidence for their corelease (Shabel et al., 2014). Despite this considerable progress, a compilation of recent studies on multitransmitter neurons (Table 1) reveals that only few examples exist to corroborate corelease or colocalization of transporters on the same vesicle (Fasano et al., 2017; Root et al., 2018; Saunders et al., 2015; Takacs et al., 2018; Zimmermann et al., 2015). Immunogold electron microscopy (IEM) following dual labeling of colocalized transporters provides indication for corelease (Takacs et al., 2018), but it is technically challenging to 
perform IEM on every cotransmitting synapses and is prone to false-negative signaling. Electrophysiological recordings of dual transmitter responses following optogenetic stimulation can indicate corelease yet, due to the nature of these techniques, they are are not conclusive and can only be applied to study specific pre-post neuronal populations (Shabel et al., 2014; Takacs et al., 2018). Functional experiments such as measuring the effect of one transmitter on the vesicular uptake of the other (Gras et al., 2008) or SV immunosisolation (Gronborg et al., 2010) goes one notch up to explore corelease of transmitters. Nonetheless, these are bulk experiments and could lead to false-positive vesicular colocalization. Thus, the above approaches, when applied either individually or together, yield a wealth of information on dual transmitter signaling but inadequate to confirm corelease and do not address the extent of the phenomenon in the whole mammalian brain.

In the present study, we have addressed these questions by visualizing different well-characterized VTs in highly purified SVs at single vesicle level, resulting in a comprehensive and quantitative colocalization map of transporters that reveals the extent and diversity of corelease in adult rat brain. We have also studied one example of corelease to understand the benefits and the relevance for synaptic physiology.

\section{RESULTS:}

\section{DyMIN STED allows high-throughput super-resolved imaging of single SVs in association with enhanced fluorescence quantum yield}

A recently developed advanced super resolution imaging technique, dynamic minimum stimulated emission depletion (DyMIN STED) nanoscopy that couples super resolution with efficient photon capture was used to image single SVs derived from rat brain. DyMIN STED uses only as much on/ off-switching light as needed to image at desired sub-diffraction resolution in contrast to conventional STED microscopy that applies constant STED laser power throughout an imaging area (Gottfert et al., 2017; Heine et al., 2017). The technique significantly reduce photobleaching, enabling efficient photon detection from single vesicles (Figure 1A-C). In contrast to conventional high-resolution microscopy (Farsi et al., 2016), DyMIN STED significantly improved resolution of single vesicles (Figure 1B). The average size of SV puncta, measured in terms of standard deviation (SD) of integrated Gaussian fit $(\sigma)$ of the fluorescence line profile across each puncta, in DyMIN STED was $~ 33 \mathrm{~nm}$ in comparison to $\sim 126 \mathrm{~nm}$ in confocal, which is analogous to the real size of SVs (Takamori et al., 2006). Additionally, adaptive STED laser intensity at detected fluorophore localizations improved signal-tonoise ratio while maintaining photon yield as comparable to that of confocal microscopy (Figure 1B). To immunolabel SVs, we developed a strategy that maximized epitope coverage while effectively removing background fluorescence from unbound antibodies (Figure 1F-H). Freshly isolated SVs of high purity (Takamori et al., 2006) were indirectly labeled using saturating concentrations of antibody 'in-solution', followed by size exclusion chromatography (SEC) to remove unbound antibodies and immobilized on glass coverslips ). SVs that are labeled by the above protocol exhibited significantly higher fluorescence intensities than SVs labeled conventionally (i.e. after immobilization on a coverslip) using the same antibody. Thus 'in-solution' labeling not only enhanced epitope coverage of SVs but is also suitable for detecting low copy number SV proteins such as transporters (Figure 1DE). Negative controls that lacked either vesicles or primary antibody exhibited no background fluorescence, allowing detection of single fluorophores (Figure S2). Thus, the combination of DyMIN STED and the labeling protocol allows high-throughput visualization of super-resolved SVs for quantitative fluorescent analysis. 


\section{Two color DyMIN STED reveals colocalization pattern of diverse transporters on the same vesicle}

To determine the proportion of SVs expressing a given transporter within the total SV population, we performed dual immunolabeling of SVs for Synaptophysin (Syp), a marker present on all SVs irrespective of the neurotransmitter phenotype ((Takamori et al., 2000), see also Figure S3), and each of the following vesicular transporters - VGLUT1, VGLUT2, VGLUT3 (specific for glutamate), VGAT (specific for GABA and Glycine), VMAT2 (specific for monoamine transmitters), VAChT (specific for acetylcholine), and ZnT3 (specific for $\mathrm{Zn}^{2+}$, Figure 2C and D). VGLUT1, VGLUT2 and VGLUT3 were detected on approximately $60 \%, 23 \%$ and $2.8 \%$ of all SVs respectively, in agreement with previous reports ((Takamori et al., 2006; Wilhelm et al., 2014), Figure 2D). VGAT labeling was detected on $15.9 \%$ of all SVs, again confirming earlier reports (Takamori et al., 2006; Takamori et al., 2000). VMAT2 and VAChT were present on $2.6 \%$ and $1.5 \%$, respectively, of the total SV population (Figure 2D). Surprisingly, ZnT3, the transporter responsible for loading $\mathrm{Zn}^{2+}$ that inhibits post synaptic AMPA and NMDA receptors (Kalappa et al., 2015; Palmiter et al., 1996; Romero-Hernandez et al., 2016) (Palmiter et al., 1996; Romero-Hernandez et al., 2016), is one of the most abundant of all VTs; a staggering $\sim 30 \%$ of all SVs were ZnT3 positive (Figure 2D). Indeed, ZnT3 protein has been consistently detected in earlier SV proteomics studies (Morceano et al., 2005, Takamori et al., 2006, Grönborg et al., 2010, Boyken et al., 2013) and also been shown to preferentially co-express at excitatory synapses when compared to inhibitory synapse (Grönborg et al., 2010, Palmiter et al., 1996). The above SV composition did not change when antibodies targeting different epitopes on the transporters were used (data not shown).

To investigate expression of multiple VTs on the same vesicle, we measured degree of colocalization between two distinct transporters in all possible pairwise combinations using dual color DyMIN STED imaging (Figure 2F-L). Colocalization was determined using a co-ordinate based colocalization analysis ((Malkusch et al., 2012), Figure S4) since SV puncta followed a Rayleigh distribution as described in previous reports ((Geumann et al., 2008), Fig S5, see Supplementary Materials for more information). For glutamatergic vesicles (excitatory), interesting differences became apparent when comparing the three VGLUT isoforms (VGLUT-1-3). First, overlap between the two abundant variants VGLUT1 and VGLUT2 was low (Fig 2F, $1 \%$ of VGLUT1 SVs contain VGLUT2) despite their coexistence during development (Herzog et al., 2006) but in agreement with non-overlapping expression pattern of the two VTs in adulthood (El Mestikawy et al., 2011; Takamori et al., 2001). Second, the VGLUT variants differ in their colocalization with other VTs. VMAT2 mainly colocalized with VGLUT2 whereas VAChT showed preference to VGLUT3 (Fig 2H), whose coexpression has been observed in dopaminergic and cholinergic neurons in midbrain and striatum (Gras et al., 2008; Hnasko et al., 2010) (Morales and Margolis, 2017). Third, VGLUT1 but not the two other variants show preferred colocalization with ZnT3 ( 37\%, Fig 2F-H).

VGAT, the transporter defining GABAergic vesicles, was shown previously to be colocalized with other VTs at many synapses (Tritsch et al., 2016). However, on the vesicle level, VGAT shows only low degree of overlap with other VTs. For instance, VMAT2 and VAChT are only found on $\sim 0.75 \%$ and $\sim 0.3 \%$ of GABAergic vesicles, respectively (Figure 2I), suggesting that corelease of GABA with monoamines or acetylcholine from the same vesicle pool is not widespread. On the contrary, both monoaminergic and cholinergic SVs generally showed higher (albeit still meagre) degrees of colocalization with VGLUTs and VGAT (Figure 2J and K, note that colocalization between VMAT2 and VAChT could not be measured, see Supplementary Materials). Finally, we also examined SVs that carry one of the VGLUT variants and VGAT. Both VGLUT1 and VGLUT2 showed similar levels 
of colocalization with VGAT (slightly below $0.5 \%$ ). Overall, VTs exhibit a diverse combination of colocalization on single SVs.

\section{SVs are highly diverse based on the phenotype of expressed vesicular transporters}

The above colocalization pattern of VTs and the broad composition of SVs carrying a specific VT allowed us to group SVs based on the phenotype of expressed vesicular transporters and quantify them (Figure 3A and B). We identified 27 classes of SVs, each carrying either one or two distinct VTs (Figure 3A). The percentage of SVs specific for a single neurotransmitter (i.e carrying only one VT) is $\sim 64$ showing that one in every four SVs is capable of storing more than one neurotransmitter. The multitransmitter SV population, however, is predominated by VGLUT1-ZnT3 vesicle, which accounts for $\sim 34 \%$ of all SVs. To validate such a high degree of colocalization between VGLUT1 and ZnT3 and the resulting abundance of VGLUT1-ZnT3 vesicles, we performed dual-label immunogold electron microscopy (IEM) of isolated SVs using VGLUT1 and ZnT3 primary antibodies and secondary species conjugated to gold nano particles (Figure 3A inset, VGLUT1-5 nm, ZnT3-10 nm). Indeed, a modest population of SVs was positive for both VGLUT1 and ZnT3, in spite of the lower sensitivity of IEM when compared to light microscopy (4.14\% of all SVs carried gold particles specific for both VGLUT1 and ZnT3; $35.6 \%$ and $6.7 \%$ of vesicles were positive for VGLUT1 and $\mathrm{ZnT3}$ alone).

We also determined the composition of excitatory SVs, characterized by the expression of either one of the three VGLUT subtypes (VGLUTs) or in combination with another VT (Figure 3C). While a variety of excitatory SVs involving different combinations of VGLUTs and other transporters exist, two defining features are very conspicuous - 1) the sheer abundance of VGLUT1-ZnT3 SVs (30\% of excitatory SVs), which we will focus in the later sections and 2) only $0.5 \%$ of excitatory SVs carry both VGLUT1 and VGLUT2. The low overlap between VGLUT1 and VGLUT2, in spite of their coexpression during early development stages (Herzog et al., 2006), reflect a near complete exchange of VGLUT1 for VGLUT2 in the adult cortex.

Inhibitory SV composition, characterized by the expression of only VGAT or in combination with another VT, has also been deduced (Figure 3D). Interestingly, only 3.5\% of VGAT positive SVs carry another transporter, which is not anticipated in view of the increasing evidence for cotransmission of GABA together with other neurotransmitters ((Tritsch et al., 2016) suggesting that VGAT and other VTs exist in segregated pools in those synapses. A very small population of GABA-Glutamatergic SVs ( $\sim 0.37 \%$ of all SVs) has also been detected carrying VGAT and one of the VGLUT subtypes. Interestingly, the composition of GABA-Glutamatergic SVs also shows some insight on VGLUT subtype that is majorly involved in GABA corelease (Fig 3E). Among the three subtypes, , VGLUT1 is preferentially colocalized with VGAT, although only VGLUT2 has so far been detected for corelease with GABA (Shabel et al., 2014) reflecting the necessity for more studies on corelease in the cortex, where VGLUT1 remain as the predominant glutamate transporter. Intriguingly, a report on glutamate and GABA corelease in the hippocampus failed to detect VGAT at the synapse raising speculation of a possible carboxylase activity to convert glutamate to GABA inside the vesicle lumen (Walker et al., 2001). However, in our comprehensive SV proteomic analysis (described in the following section), no enzymes required for this conversion was detected (Table S2).

Taken together, the diversity of synaptic vesicles carrying two VTs was surprising. Conversely, the overall degree of colocalization between any transporter pair is low, being consistently below $5 \%$. Thus, a vast majority of all SVs are indeed specific for only a single neurotransmitter, with an exception of VGLUT1-ZnT3 SVs. 


\section{ZnT3 is a highly abundant SV protein specifically enriched in VGLUT1 positive vesicles}

To verify the high abundance of VGLUT1-ZnT3 SVs, we conducted a series of quantitative investigations to deduce ZnT3 protein abundance, expression and distribution. First, we measured the amount of ZnT3 protein in SVs using quantitative immunoblotting using highly purified ZnT3 protein $(42 \mathrm{kDa})$ as standard (Figure 4A). Increasing amounts of SVs and purified ZnT3 was loaded on the same gel and ZnT3 was detected using a specific monoclonal antibody (Figure 4A). The amount ZnT3 in SVs, obtained from the standard curve, was $\sim 12 \mathrm{ng} / \mu \mathrm{g}$ of SVs of SV protein $(\mathrm{n}=2,261.90 \pm 23$ $\mathrm{fmol} / \mu \mathrm{g}$ of SVs), which corresponds to $\sim 1.5 \%$ of total SV protein (Figure 4B, see (Takamori et al., 2006). Next, we performed quantitative mass spectrometry (MS) of SV proteome using purified SVs resulting in comprehensive detection of $\sim 1500$ proteins each with individual IBAQ values (Figure 4C, Table S2). The detected proteins included standard SV markers such as synaptophysin, synaptobrevin2 and synaptotagmin-2 in established stoichiometry and all the transporters that are investigated in the current study. In agreement with immunoblotting measurements, the amount of ZnT3 measured by MS was $155 \pm 43 \mathrm{fmol} / \mu \mathrm{g}$ of SV protein, which is roughly one-third of VGLUT 1 amount (Figure 4C).

The neurotransmitter content of SVs depends on the copy number of transporter molecules/vesicle (Pothos et al., 2000). To determine how the absolute amount of ZnT3 translates into copy numbers, we developed an imaging based approach using DyMIN STED (Figure 5A). Isolated SVs were labeled for VGLUT1 or ZnT3 using primary monoclonal antibody and secondary nanobody (Sograte-Idrissi et al., 2020) conjugated to a definite number of fluorophores resulting in a fixed stoichiometry between the number of protein molecules and the fluorophores in single vesicles. Copy number of proteins in individual SVs was obtained by dividing the SV fluorescent intensity with the mean intensity of free antibody-nanobody complex (Figure 5A). Compared to conventional estimation of copy number that relies on bulk measurements yielding only an average value (Taoufiq et al., 2020), the imaging based approach revealed variability of copy number between vesicles. The copy numbers of both VGLUT1 and ZnT3 were quite variable with an average of $\sim 8$ and $\sim 3.5$ copies/SV, respectively (Figure 5B). Finally, to corroborate colocalization of with VGLUT1, we performed immunoisolation of ZnT3 SVs using monoclonal anti-ZnT3 antibody and detected selected proteins through western blotting. ZnT3 immunoisolated SVs showed, as expected, selective enrichment of VGLUT1 when compared to the control SVs that were immunoisolated using synaptobrevin2 antibody representing all SVs (Figure S8). In addition to VGLUT1, another SV membrane protein, SV2B was also enriched in ZnT3 sample concurring with earlier findings in SVs immunoisolated with VGLUT1 antibodies (Gronborg et al., 2010). Overall, the above data reveals that ZnT3 is an abundant integral component of VGLUT1containing SVs.

\section{Heterogeneous pools of SVs containing VGLUT1, ZnT3 and VGLUT1-ZnT3 reside at a single synapse}

VGLUT1 and ZnT3 are known to exhibit overlapping expression pattern ((McAllister and Dyck, 2017). To compare the synaptic distribution of VGLUT1 and ZnT3, we labeled the transporters together with an active zone marker, Piccolo, in cultured primary hippocampal neurons and visualized them using confocal microscopy. A pixel based correlation revealed a high degree of overlap between between both transporters (Figure S6B and C) indicating that almost every VGLUT1 synapse is also positive for ZnT3. To further probe the intrasynaptic distribution of VGLUT1 and ZnT3, we imaged culture neurons at single vesicle resolution using DyMIN STED following immunolabeling of the transporters in separate experiments (Figure 5C-F). The density of ZnT3 positive SVs at individual 
boutons was smaller than that of VGLUT1 positive SVs (Figure 5F). Interestingly, ZnT3 positive SVs appear to be more proximal to the active zone than VGLUT1 SVs indicating presence of spatially segregated pools of SVs containing - only VGLUT1, only ZnT3 and VGLUT1-ZnT3 at the synapse. This microheterogeneity of SV pools might reflect functional relevance such as enhanced calcium sensitivity as observed in ZnT3 wildtype mice when compared to the knockout (Lavoie et al., 2011), or differential recycling routes between the pools (Figure $5 \mathrm{G}$ ).

\section{$\mathrm{Zn}^{2+}$ facilitates vesicular glutamate uptake by a mechanism mediated by ZnT3}

Does the presence of an active $\mathrm{Zn}^{2+}$-transport affect VGLUT1 transport activity? To address this issue, we measured glutamate uptake in enriched SV fractions (LP2, (Preobraschenski et al., 2014)) using purified protein-based glutamate sensor, iGluSnFR (Marvin et al., 2018) in the presence of different

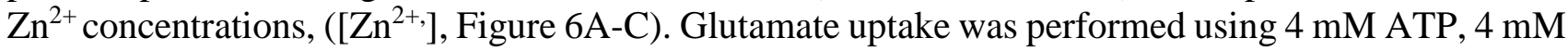
$\mathrm{KCl}, 10 \mathrm{mM}$ potassium glutamate in MOPS-Glycine uptake buffer in the presence or absence of proton ionophore, FCCP and high affinity specific $\mathrm{Zn}^{2+}$ chelator, TPEN. Data were corrected for uptake in the presence of FCCP and normalized to the TPEN condition. Glutamate uptake into vesicles was dose-dependently facilitated by $\mathrm{Zn}^{2+}$ and exhibited a $\sim 2$ fold increase in the maximal uptake with an EC50 of $\sim 25 \mathrm{nM}$ (Figure $6 \mathrm{~B}$ and C), a physiologically relevant cytoplasmic $\left[\mathrm{Zn}^{2+}\right]$ in view of the confined volume of a typical presynaptic compartment $\left(3.7 \times 10^{-22} \mathrm{~L}\right.$, (Goch and Bal, 2020; Wilhelm et al., 2014)) and activity-dependent increase of cytoplasmic [ $\left.\mathrm{Zn}^{2+}\right]$ (Sanford et al., 2019). To determine if the facilitatory $\mathrm{Zn}^{2+}$ effect on VGLUT1 activity is mediated by ZnT3, we performed the glutamate uptake assay in preparations from ZnT3 knockout (KO) mice. Although KO mice showed no apparent change in VGLUT1 expression level (Figure S8), the basal glutamate uptake performed in the presence of $1 \mathrm{pM} \mathrm{Zn}^{2+}$ was slightly reduced ( 20\%, Figure 6A) suggesting that ZnT3 is involved in the determination of glutamate quantal size in resting conditions concurring with the reduction in the amplitude of miniature and spontaneous excitatory postsynaptic currents observed in ZnT3 KO mice (Lavoie et al., 2011). Moreover, the facilitatory effect of $\mathrm{Zn}^{2+}$ on glutamate uptake was completely abolished in $\mathrm{KO}$ implying the existence of an activity-dependent regulation of vesicular glutamate content by $\mathrm{Zn}^{2+}$ through $\mathrm{ZnT3}$ (Figure 6B).

\section{Ambient $\mathrm{Zn}^{2+}$ increases synaptic quantal size via $\mathrm{ZnT3}$}

To determine if the facilitatory effect of $\mathrm{Zn}^{2+}$ on vesicular glutamate uptake lead to enhanced quantal glutamate release, we explored if $\mathrm{Zn}^{2+}$ affects miniature excitatory postsynaptic currents (mEPSCs) that represents synaptic quantal release of glutamate. We performed whole-cell recordings in hippocampal CA3 pyramidal neurons that receives signals from mossy fiber terminals that are enriched in $\mathrm{Zn}^{2+}$ containing vesicles. AMPAR mediated mEPSCs were obtained by in the presence of TTX, bicuculline and D-APV in ZnT3 wildtype (WT) and knockout (KO) mice, As reported previously (Lavoie et al., 2011), the mEPSC peak amplitude and frequency was decreased in ZnT3 KO animals when compared to the WT (Figure 7B-D). Interestingly, the level of reduction in the peak amplitude $(\sim 20 \%)$ is comparable to the magnitude of reduction in glutamate uptake in ZnT3 KO SVs (see Figure 6A) suggesting that ZnT3 mediated facilitation of vesicular glutamate uptake underlies basal synaptic quantal size in the WT. . Next, to determine if $\mathrm{Zn}^{2+}$ mediated facilitation of vesicular glutamate uptake lead to enhanced quantal size, we explored the effect of exogenous application of $\mathrm{Zn}^{2+}$, which results in increased cytoplasmic $\mathrm{Zn}^{2+}$ concentration (Sanford et al., 2019), on quantal properties. We measured mEPSCs in the presence of $20 \mu \mathrm{M}$ of $\mathrm{Zn}^{2+}$ in the bath solution following a baseline recording. There was no change in the mEPSCs following $\mathrm{Zn}^{2+}$ application $\left(\mathrm{n}=8\right.$, data not shown). Since $\mathrm{Zn}^{2+}$ mediated facilitation of glutamate uptake is dose dependent, we performed the above experiment in higher $\mathrm{Zn}^{2+}$ 
concentration. In the presence of $200 \mu \mathrm{M}$ of $\mathrm{Zn}^{2+}$, the mEPSC peak amplitude was significantly increased in the WT (Figure 7B-D) indicating $\mathrm{Zn}^{2+}$ mediated regulation of glutamatergic quantal size. However, there was no change in the frequency, decay and rise time kinetics indicating that ambient $\mathrm{Zn}^{2+}$ did not alter release probability or induce postsynaptic changes. Finally, we tested the effect of ambient $\mathrm{Zn}^{2+}$ on neurotransmission in $\mathrm{ZnT} 3 \mathrm{KO}$ mice. Following $\mathrm{Zn}^{2+}$ application, there was no change in the mEPSC amplitude or frequency (Figure 7B-D) in the KO indicating that the increase in glutamate quantal size is mediated via ZnT3. Overall, the above data demonstrate dose-dependent regulation of glutamatergic neurotransmission by enhancing synaptic quantal size via ZnT3 co-present on VGLUT1 SVs. 


\section{DISCUSSION}

\section{Corelease of multiple neurotransmitters from the same vesicle}

Release of multiple neurotransmitters from the same neuron or synapse is well documented (El Mestikawy et al., 2011; Granger et al., 2017; Hnasko and Edwards, 2012). Here, we report quantitative insights on corelease of transmitters from the same vesicle and its impact on synaptic function. Using an advanced super resolution imaging in combination with robust labeling strategy and electron microscopy, we show prevalence of corelease by quantifying diverse SVs based on transporter phenotype. Next, we validated the unexpected abundance of VGLUT1-ZnT3 SVs using quantitative biochemical and mass spectrometry analysis. Finally, using electrophysiological recordings and in vitro experiments, we demonstrate physiological implications of transporter colocalization on synaptic function. We show that 1) nearly $\sim 36 \%$ of all synaptic vesicles carry two vesicular transporters and thus capable of storing and releasing distinct neurotransmitters, 2) however, the SV type containing VGLUT1 and ZnT3 exceedingly accounts for the majority of multitransporter vesicles ( $34 \%$ of all SVs), 3) the multitransporter SVs are highly diverse than anticipated suggesting corelease of all combinations of dual neurotransmitters, 4) colocalization of VGLUT1 and ZnT3 increases vesicular glutamate content leading to enhanced post synaptic response. We propose that that localization of distinct VTs may underlie a specific mechanism to regulate transmitter quantal size and thus synaptic function.

Since only $\sim 2 \%$ of all SVs excluding those containing VGLUT1 contain distinct SVs, we conclude that the majority of neuronal SVs are specific for only one neurotransmitter. Nonetheless, the unexpected combinatorial diversity of colocalized VTs reveals a new dimension of synaptic finetuning that extends well beyond the hitherto documented cases of cotransmission. Although the colocalization pattern of transporters discovered in this study supports, in large, existing knowledge, the data reveals few surprising combinations as well. For example, glutamate-GABA corelease has been mostly observed at synapses containing VGLUT2 and VGAT (Root et al., 2014; Shabel et al., 2014). However, our data shows that both VGLUT1 and VGLUT2 exhibit similar levels of colocalization with VGAT. This discrepancy arises, perhaps, because VGLUT1 expressing cortical synapses have not yet been extensively studied for corelease. The findings also reveal that only a small fraction of VGAT positive SVs express another transporter although increasing evidence suggests widespread incidences of cotransmission of GABA with other transmitters. This could either reflect presence of segregated pools of SVs containing GABA and other transmitters at the reported synapses or such synapses constitute only a small component of the whole inhibitory circuit.

It should be noted that mere localization of multiple VTs on individual SVs does not imply corelease because it depends on the expression of the corresponding postsynaptic receptors and neurotransmitter synthesizing enzymes as well. Therefore, we asked if this vesicular infrastructure could underlie a specific presynaptic function by means of regulating VT activity. This prospect was presented earlier for VAChT and VGLUT3 or VMAT2 and VGLUT3, where glutamate was shown to "synergistically" increase acetylcholine or dopamine accumulation (Gras et al., 2008; Hnasko et al., 2010) but a direct evidence that both the transporters being present on the same vesicle was lacking. We report direct colocalization of two different transporters, VGLUT1 and ZnT3, in a large population of SVs and demonstrate that one of the transporters facilitate the activity of the other leading to quantal changes of neurotransmitter release. Although such a high degree of colocalization is surprising, it is consistent with the detection of ZnT3 in VGLUT1 positive excitatory synapses (McAllister and Dyck, 2017) and selective enrichment in VGLUT1 immunoisolated SVs (Gronborg et al., 2010; Salazar et al., 2005). 
Moreover, co-targeting of VGLUT1 and ZnT3 to the same destination vesicle following heterologous expression has been previously shown in PC12 cells (Salazar et al., 2005). This also supports earlier findings of temporally coinciding release of $\mathrm{Zn}^{2+}$ and glutamate at specific synapses (Lavoie et al., 2011; Vergnano et al., 2014). Together, the prevalence of VGLUT1 and ZT3 coexpressing vesicles and the influence on quantal glutamate underlies a widespread and novel regulatory mechanism of systemic synaptic excitation.

\section{Synaptic and circuit function implications of high overlap between VGLUT1 and ZnT3}

Corelease of neurotransmitters from same SV pools has been demonstrated in handful of synapses but the effect on synaptic computation and circuit function has been addressed only in fewer synapse (Liu et al., 2013; Shabel et al., 2014). Since functional implications of corelease of distinct transmitters depend on various factors including the identity of neurotransmitters, localization of pre- and postsynaptic receptors and activity-dependent changes, if any, in all of these factors, a generalized scheme of synaptic signaling is difficult to achieve. However, a common feature can be hypothesized for SVs specific for particular dual transmitters. For VGLUT1-ZnT3, the effect of the dual transmitters, glutamate and $\mathrm{Zn}^{2+}$, on the postsynaptic receptors is fairly known; they both bind postsynaptic AMPA and NMDARs. Intriguingly, they exert antagonistic function, with glutamate exhibiting an excitatory response on postsynaptic neurons while $\mathrm{Zn}^{2+}$ inhibiting the same (Kalappa et al., 2015; Vergnano et al., 2014) indicating that $\mathrm{Zn}^{2+}$ might primarily act to fine tune the excitation window, as observed in glutamate-GABA coreleasing neurons (Shabel et al., 2014). Considering the high abundance of VGLUT1-ZnT3 SVs from single vesicle imaging experiments, $\mathrm{Zn}^{2+}$ action could underlie a systemic and robust mechanism to modulate excitation. When compared to the inhibitory circuit, mediated by GABA that predominantly balances excitation through a separate population of inhibitory neurons, $\mathrm{Zn}^{2+}$ is spatio-temporally locked with glutamate release to modulate excitation. Indeed, synaptic zinc, released by short trains of stimuli, selectively inhibits postsynaptic GluN2A-NMDARs and causes changes in synaptic integration and plasticity (Vergnano et al., 2014). It is, however, highly conceivable that such a direct effect depends on the magnitude of synaptic $\mathrm{Zn}^{2+}$ at a particular synapse, which relies on the expression level/copy number of ZnT3 in individual SVs. As the copy number ZnT3, as determined in the present study, is highly heterogeneous (between 1 -8), a region-specific zinc action is more plausible depending the expression level of ZnT3.

One prominent feature of the above mechanism is that $\mathrm{Zn}^{2+}$, unlike other neurotransmitters, is not synthesized by an enzymatic machinery but its cytoplasmic concentration is regulated by neuronal activity (Minckley et al., 2019; Sanford et al., 2019), which could lead to activity-dependent regulation of quantal glutamate mediated by ZnT3. To our knowledge, this is the first evidence of a direct role in basal synaptic mechanism by $\mathrm{Zn}^{2+}$, the dishomeostasis of which has been implicated in several neurodegenerative conditions and synaptic pathologies (Frederickson et al., 2005; Kawahara et al., 2020; Trist et al., 2018).

What will be the physiological consequence of this facilitation at the circuit level? Tzounopoulos and colleagues recently demonstrated that synaptically released zinc reduce the gain of sound-evoked responses in primary auditory cortex, A1 (Anderson et al., 2017). Although this is anticipated due to the inhibitory effect of zinc on glutamatergic receptors, the gain of principal and specific inhibitory neurons' responses were shown to be differentially affected requiring clarity on the mechanism of zinc action. Based on our results, one potential explanation could be that synaptic zinc, by enhancing vesicular glutamate uptake, increases net glutamatergic drive in the circuit that specifically increased feedforward inhibition resulting in a decline in the overall gain of A1. One way to test this hypothesis would be to directly visualizing glutamate release in vivo following expression of glutamate sensors 
such as iGluSnFR. As gain modulation is increasingly shown to control the dynamic range of neuronal responses to sounds and other sensory inputs (Olsen et al., 2012), earlier observed changes in synaptic zinc in vivo during sensory experience suggest a (Brown and Dyck, 2002; Dyck et al., 2003)profound link between activity-dependent changes in synaptic zinc and sensory processing

\section{Mechanism of glutamate and $\mathrm{Zn}^{2+}$ dual uptake}

The mechanism of zinc action on glutamate uptake is yet to be investigated. However, the existing knowledge on the transport mechanism of glutamate and zinc uptake through VGLUT1 and ZnT3, respectively, could shed light on the mechanistic understanding of the dual transmitter uptake. Accumulation of most of the neurotransmitters in the vesicle lumen is regulated by a coordinated activity of vacuolar proton ATPase (V-ATPase) and VTs. The activity of V-ATPase, which is universally present in all types of vesicles independent of the neurotransmitter stored, drives protons into the lumen, generating a proton electrochemical gradient $\left(\Delta \mu \mathrm{H}^{+}\right)$. The VTs, which are exclusive to specific vesicle types, then use the electrochemical gradient to drive neurotransmitter transport against a concentration gradient. As different VTs use the chemical $(\Delta \mathrm{pH})$ and electrical $(\Delta \Psi)$ components with varied efficiencies, regulation of the two components critically influence neurotransmitter uptake (Edwards, 2007). VGLUTs are thought to be predominantly driven by membrane potential (Juge et al., 2010; Juge et al., 2006), although a growing body of evidence indicate participation of $\Delta \mathrm{pH}$ as well (Bellocchio et al., 2000; Schenck et al., 2009). While functional studies involving liposome reconstitution or single SV imaging propose VGLUT1 as a $\mathrm{K}^{+} / \mathrm{H}^{+}$exchanger (Farsi et al., 2016; Preobraschenski et al., 2014), electrophysiological recordings imply an allosteric activation of VGLUT by protons from the luminal side (Eriksen et al., 2016). Indeed, a recent cryo-electron microscopy (Cryo-EM) structure of VGLUT2 elucidates a structure-based transport mechanism that implicate both $\Delta \mathrm{pH}$ and $\Delta \Psi$ (Li et al., 2020). Another characteristic feature of VGLUTs is their biphasic dependence on chloride. While low concentration of chloride $(4 \mathrm{mM})$ is required for the transport activity of VGLUTs, absence or high concentrations of chloride substantially reduces glutamate transport. The stimulatory effect of chloride has been attributed to an allosteric activation of the transporter at low concentration (Preobraschenski et al., 2018) and, at high concentrations, chloride seems to dissipate the membrane potential and thus reduces the driving force for glutamate uptake (Preobraschenski et al., 2018; Schenck et al., 2009). A direct interaction of chloride with VGLUT and thus competing with glutamate to exert its inhibitory effect at high concentrations has been shown by many studies (Bellocchio et al., 2000; Preobraschenski et al., 2018; Schenck et al., 2009). However, arguments counter to this notion also exist (Juge et al., 2010; Juge et al., 2006). Considering the above complexity, the facilitatory effect of $\mathrm{Zn}^{2+}$ discovered in the current study renders additional complexity to the mechanism of glutamate transport. $\mathrm{Zn}^{2+}$ transport is mediated by $\mathrm{ZnT3}$ which belongs to SLC30 family of $\mathrm{Zn}^{2+}$ transporters known for $\mathrm{Zn}^{2+/} \mathrm{H}^{+}$exchange (Eide, 2006) and thus vesicular $\mathrm{Zn}^{2+}$ transport is expected to directly affect $\Delta \mathrm{pH}$. Furthermore, being a divalent cation, $\mathrm{Zn}^{2+}$ is poised to a play a major role in the determination of luminal membrane potential (Goh et al., 2011). Indeed, a recent Cryo-EM structure of human $\mathrm{ZnT}$, a close family member of ZnT3, elucidates exchange of two $\mathrm{Zn}^{2+}$ ions for two protons resulting a net increase of two positive charge in the lumen in every $\mathrm{Zn}^{2+}$ transport cycle (Xue et al., 2020). Thus, by lowering $\Delta \mathrm{pH}$ and increasing $\Delta \Psi, \mathrm{Zn}^{2+}$ transport can conveniently support vesicular glutamate uptake. Finally, it is also quite possible that $\mathrm{Zn}^{2+}$ coordinates with glutamate in the vesicle lumen resulting in reduced active glutamate amount and osmotic pressure across the membrane, which will potentially increase the upper limit of vesicular glutamate concentration (Krezel and Maret, 2016). Such a mechanism is well established for the storage of insulin in pancreatic beta-cell granules where two $\mathrm{Zn}^{2+}$ ions coordinates six insulin monomers. To understand glutamate and zinc dual transport and the facilitatory effect of $\mathrm{Zn}^{2+}$ on glutamate uptake, rigorous investigations are required to quantify vesicular $\mathrm{Zn}^{2+}$ concentration and the transport mechanism of 
ZnT3. The availability of highly purified ZnT3 should allow functional reconstitution in liposomes or hybrid vesicles (Preobraschenski et al., 2018) to study SV $\mathrm{Zn}^{2+}$ transport in the future. Furthermore, one can perform vesicular $\mathrm{Zn}^{2+}$ uptake assay as performed in the current study for glutamate to directly measure concentration of $\mathrm{Zn}^{2+}$ in $\mathrm{SV}$ s using a specific $\mathrm{Zn}^{2+}$ sensor. Overall, the new findings draw striking similarity between regulation of substrate uptake by $\mathrm{Zn}^{2+}$ in synaptic vesicles and the wellknown $\mathrm{Zn}^{2+}$ mediated insulin uptake in secretory vesicles via $\mathrm{ZnT} 8$ in pancreatic beta cells. Disruption of ZnT8 activity is a major cause of Type-I diabetes leading to enhanced therapeutic efforts targeting ZnT8 (Pociot and Lernmark, 2016). It has be to be seen if acute disruption of ZnT3 activity underlies glutamate or metal ion dishomeostasis, which is a hallmark of major neurological diseases (Frederickson et al., 2005).

\section{Limitations of the study}

We evaluated colocalization of all major vesicular transporters, which have been well studied and possess specific antibodies against them. Minor contribution from transporters that are not yet well characterized such as VNUT has not been accessed. Colocalization of more than two transporters on single vesicles was not examined in the present study. Although, it is technically feasible to perform three color DyMIN STED acquisition, epitope coverage by three different antibodies may not be optimal which could lead to false-negative colocalizations. Developments of specific nanobodies, which are one-tenth of IgG size, for the transporters would help future studies to examine localization of more than two transporters on single vesicles. Additional and synapse-specific studies are required to clarify, whether in every single case, colocalization of VTs signifies corelease of the respective transmitters and to which extent there is cross-talk between different VTs during vesicle loading. Moreover, the vesicular colocalization profile discovered in this study applies to the whole brain and, hence, the quantification could be very different in specific regions. 
ACKNOWLEDGEMENTS: We thank Stefan Hell for providing guidance and resources for imaging experiments. We thank members of Jahn lab for useful discussions, Raza-Ur Rahman for RSeq analysis (Broad Institute), Brigitte Barg-Kues for genotyping and Sigrid Schmidt for preparing culture neurons. We thank Silvio Rizzoli (Göttingen), Gudrun Ahnert-Hilger (Berlin), Pablo Castillo (Ney York) and Agata Witkowska (Berlin) for helpful comments on the manuscript. This research project was conducted with support from Volkswagen Foundation ('Experiment' grant to SS), European Research Council Advanced Grant (SVNeuroTrans to RJ) the John Black Charitable Foundation (to IM) and Deutsche Forschungsgemeinschaft (SFB1286 to HU)

\section{AUTHOR CONTRIBUTIONS}

SS conceptualized the study, performed experiments, evaluated all data and wrote the paper. RJ provided resources, evaluated data and edited the paper. NU, VM, JP, MG, LB, and EZ designed and performed different aspects of the study. JJ and IM performed and analyzed electrophysiology experiments. MN, IS and HU performed mass spectrometry studies and analyzed data. AP drafted and wrote the imaging analysis workflow. DR performed electron microscopy and analyzed data.

\section{DECLARATIONS OF INTERESTS}

Authors declare that they have no competing interests. 
A
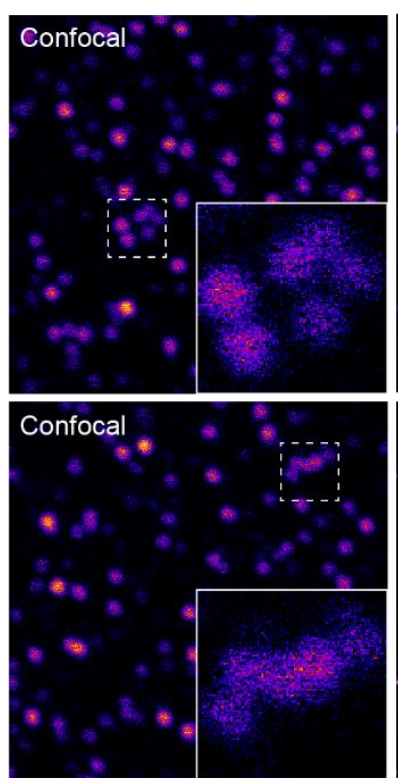

0

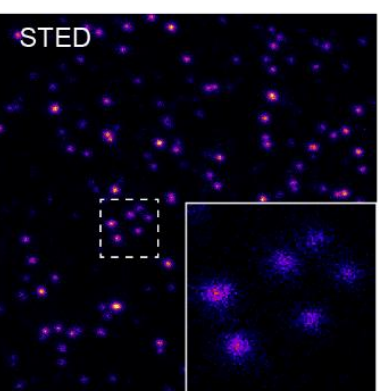

\section{Dymin}

\section{STED}
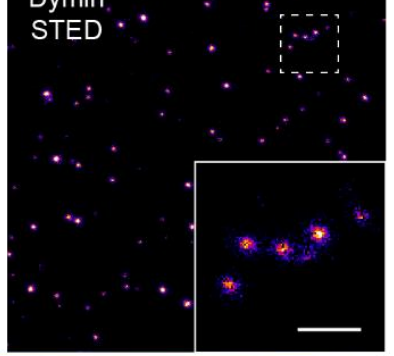

counts
B
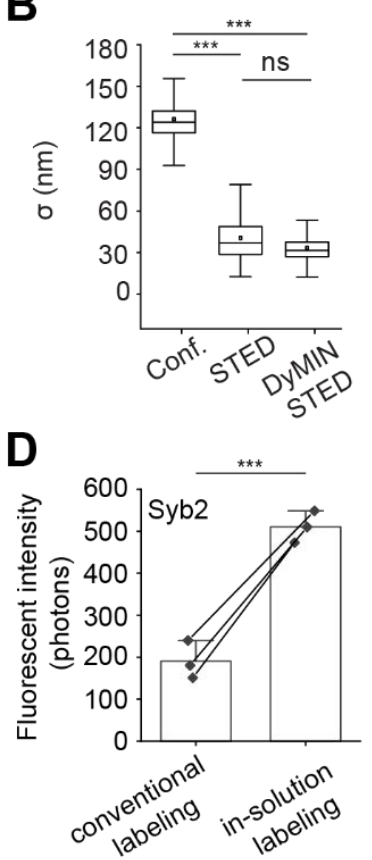
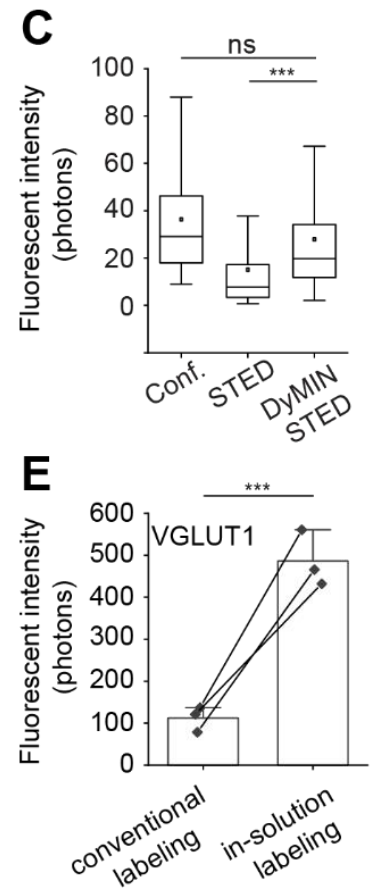

$\mathbf{F}$
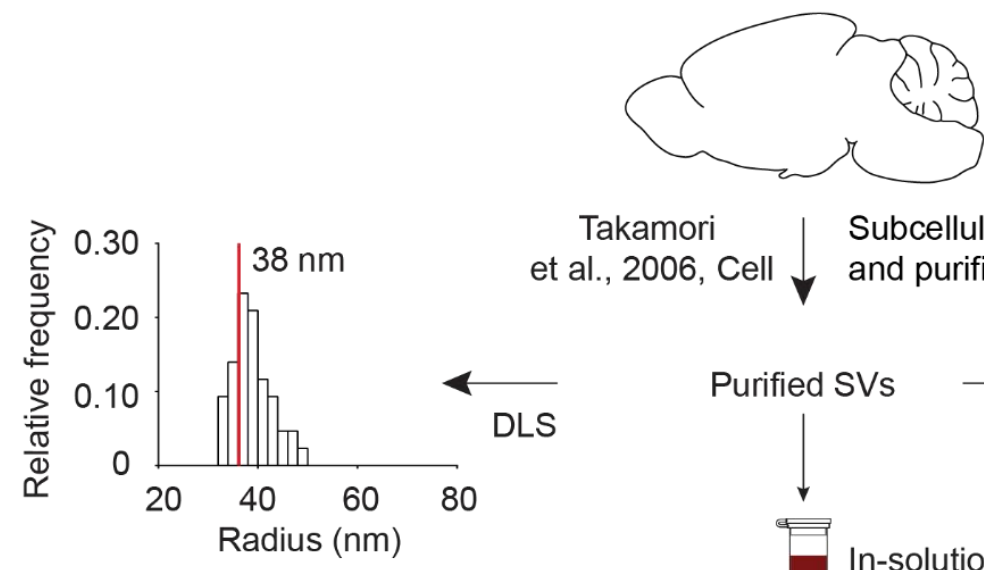

Takamori et al., 2006, Cell

Subcellular fractionation

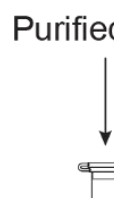

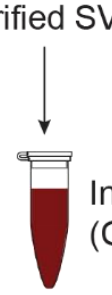

H

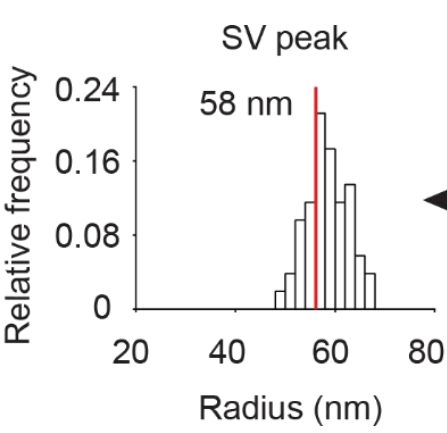

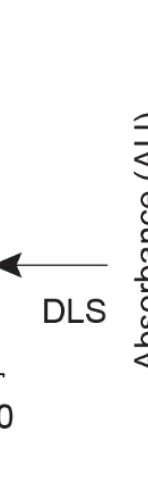

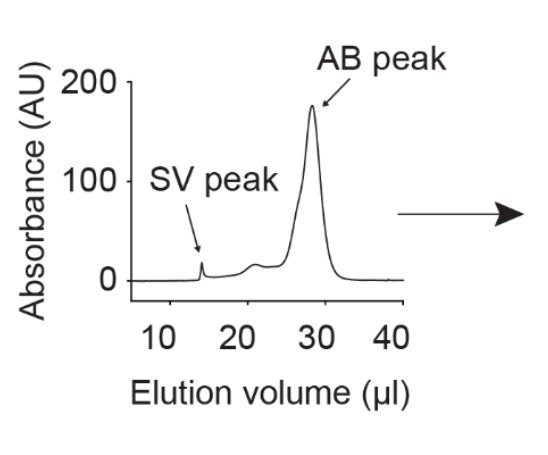

SEC purification and purification

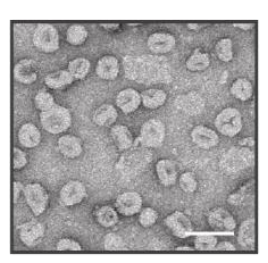

In-solution immunolabeling

(Conventional / Multiplexing)

SV peak

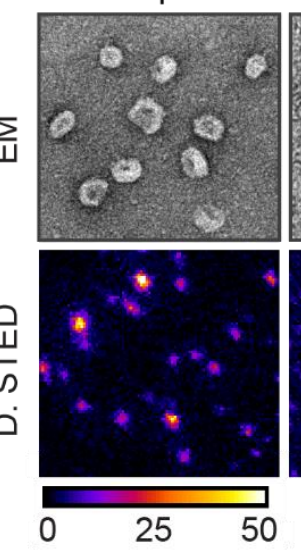

AB peak

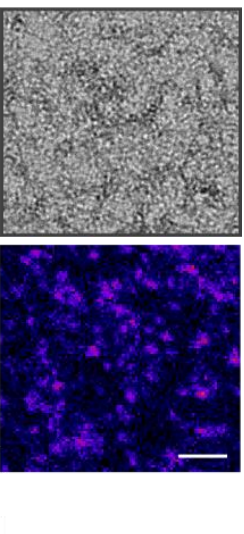




\section{Figure 1. DyMIN STED allows high-throughput super-resolved imaging of 'in-solution' labeled single SVs in association with enhanced fluorescence quantum yield}

(A) Representative images of labeled SVs (Syb2) in confocal, conventional- and DyMIN STED mode for comparison. Insets are magnified regions marked in dashed white squares showing putative single vesicles in confocal that are clearly resolved into multiple vesicles in the conventional- and DyMIN STED. (B) Box plot showing a significant increase in the spatial resolution of single vesicles in the conventional- and DyMIN STED mode in comparison to confocal mode $(* * * \mathrm{P}<0.01$; Wilcoxon signed-rank test, $n=3$ ). (C) Box plot showing comparable fluorescent intensity of individual vesicles between confocal and DyMIN STED indicating efficient photon yield (reduced photobleaching) in contrast to conventional STED ( $\mathrm{p}=1.74$, ns, between confocal and DyMIN STED; $* * * \mathrm{P}<0.01$, between conventional and DyMIN STED; unpaired $t$-test; $\mathrm{n}=3$ ). (D and E) Bar graphs showing significant increase in fluorescent intensity when vesicles were labeled 'in-solution' (as shown in F-H) in comparison to conventional labeling $(* * * \mathrm{P}<0.01$; paired $t$-test, $\mathrm{n}=3)$. Note that there is $\sim 5$ fold increase in the fluorescence intensity when SVs are labeled for VGLUT1 compared to $\sim 2.5$ fold increase for Syb2, indicating enhanced epitope coverage for a low copy number protein such as VGLUT1. (F-H) Overview of the workflow of synaptic vesicle isolation, labeling and further purification for DyMIN STED single vesicle imaging. (F) Purified SVs were prepared by a multi step subcellular fractionation, as described in Takamori et al. The purity and homogeneity of isolated SVs was tested using electron microscopy (EM) and diffraction light scattering (DLS). The mean size of individual SVs, as measured by DLS particle radii was $\sim 38 \mathrm{~nm}(\mathrm{n}=22$ experiments). DLS particle count was used as a measure for concentration of individual vesicles in the further steps. (G) After a blocking step, isolated SVs were immunolabeled 'in-solution' using saturating antibody concentrations of SVs followed by size exclusion chromatography (SEC) to separate unbound free antibodies. (H) Representative chromatogram (middle) demonstrates clear separation between the labeled SVs (SV peak) that is eluted in void volume and other contaminants such as free antibodies and serum proteins present in the blocking solution (AB peak). The quality of SEC purification was verified by subjecting the SV and $\mathrm{AB}$ peaks for DLS measurements in all experiments (left). Note that the mean size of SVs (red line in the DLS histogram) is increased $(\sim 20 \mathrm{~nm})$ following immunolabeling indicating the change in size of SVs due to the bound antibodies. DLS measurement was not possible in the AB peak due to the presence of non-homogenous particles. In some experiments, EM and DyMIN STED was used to examine SV and AB peaks following direct immunolabeling (right). As expected, only the SV peak contained detectable SVs in EM, whereas fluorescence was detected in both the samples. The labeled SVs were then immobilized on glass coverslips to prepare for DyMIN STED. All values are means \pm SEM. Scale bars in A=500 nm; $(F)=50 \mathrm{~nm} ;(\mathrm{H})=100 \mathrm{~nm}$ 

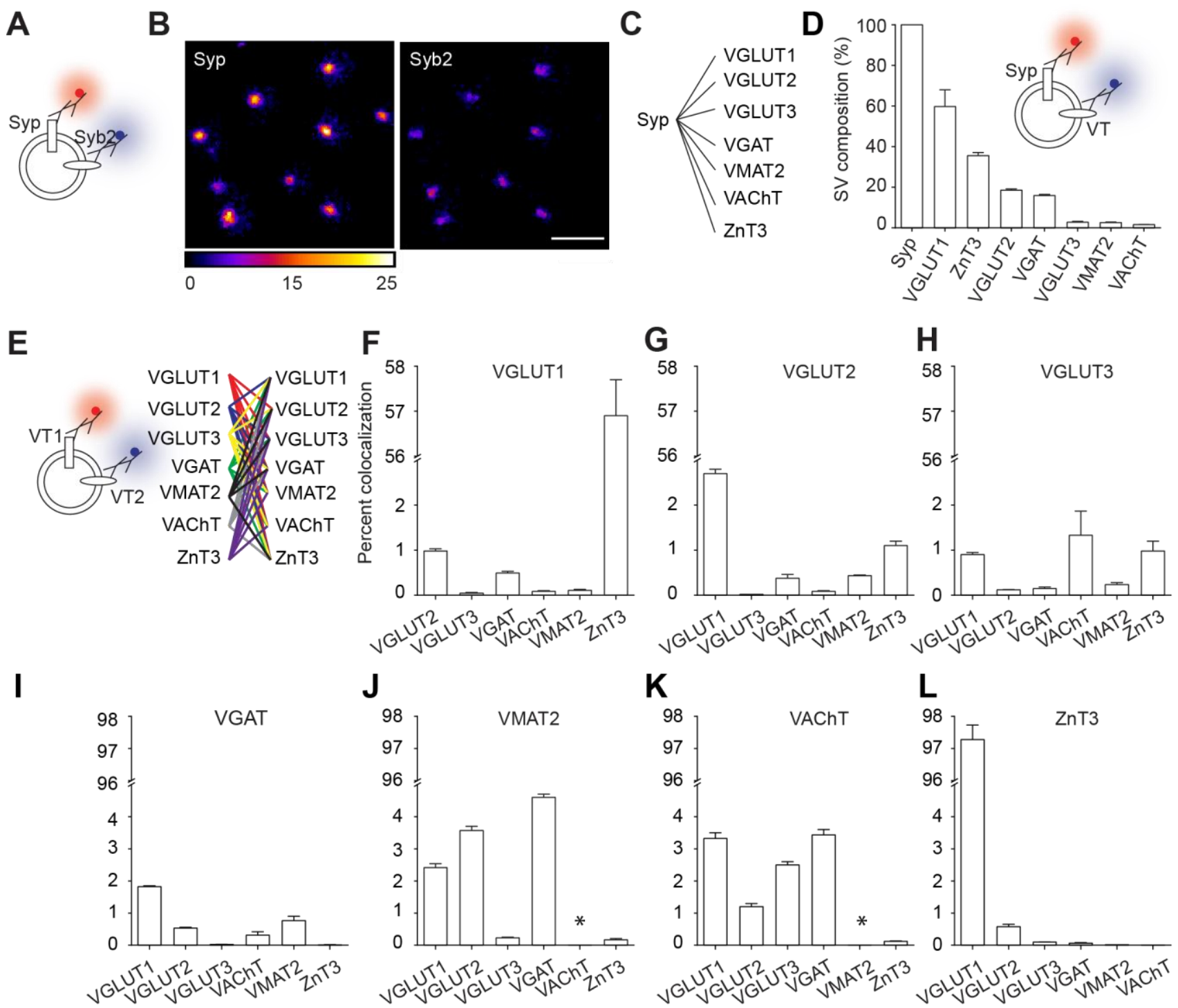

Figure 2. Two color DyMIN STED reveals colocalization pattern of diverse transporters on the same vesicle

(A) Illustration depicting double immunolabeling of SVs against Syp and Syb2 using two spectrally distinct fluorescent dyes. (B) Representative dual color DyMIN STED images showing single vesicles labeled for Syp and Syb2. (C) Schematic showing dual immunolabeling of SVs against Syp (reference for all SVs) and different vesicular transporters (VTs). (D) Bar graph quantifying the proportion of SVs expressing a specific VT. The inset illustrates double immunolabeling of SVs against Syp and a VT. (E) Left, illustration depicting dual immunolabeling of SVs against two different VTs (VT1 and VT2). Right, schematic showing all types of dual labeling experiments shown in F-L. (F-L) Bar graphs showing the degree of vesicular colocalization of a specific VT indicated on top of individual graphs against the VTs on the X-axis. No data are shown for VMAT2 and VAChT combination. Each pair of dual labeling experiments (D, F-L) was performed at least three times. All values are means \pm SEM. Scale bar in $(B)=500 \mathrm{~nm}$. 
A

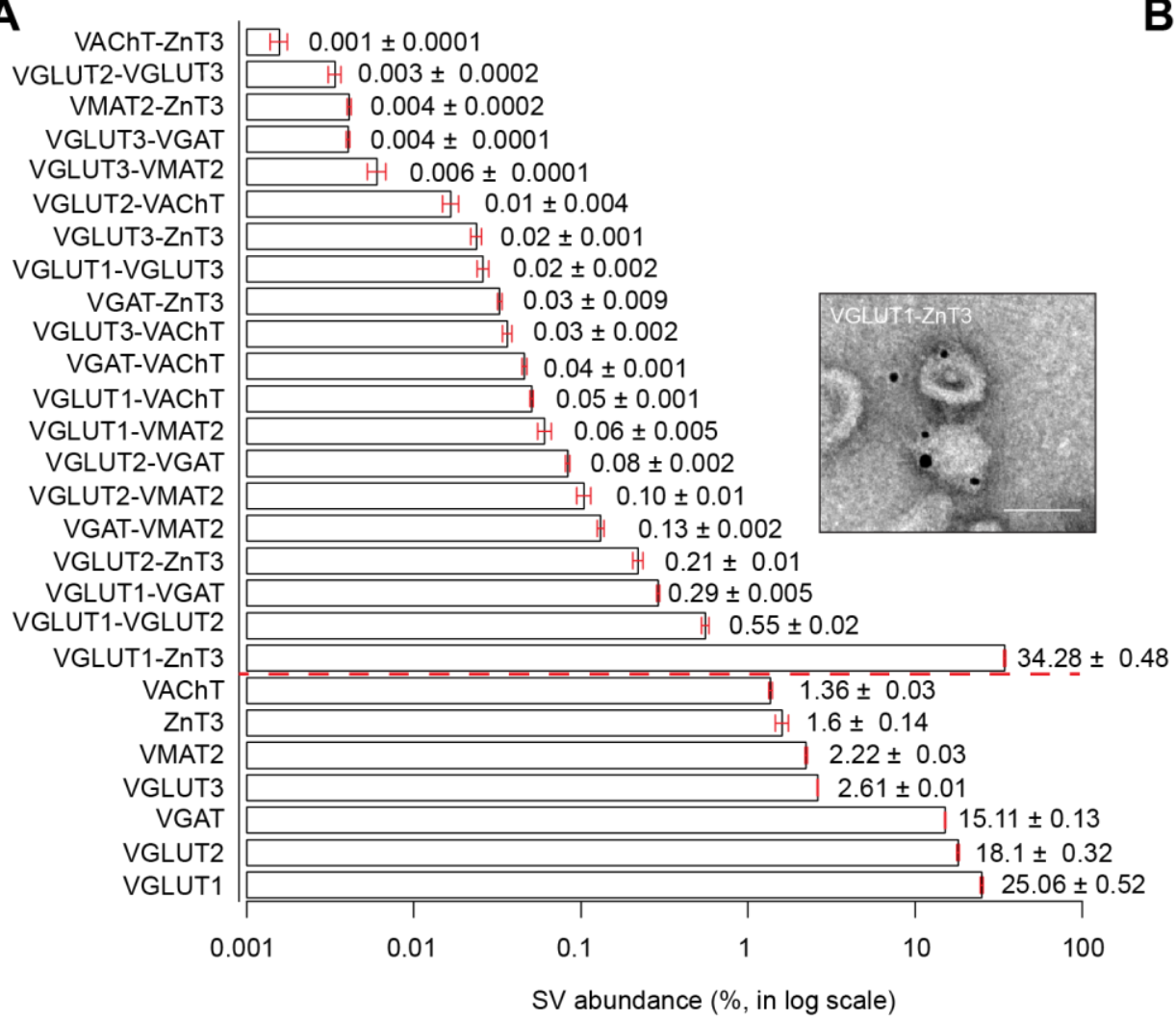

Total SV composition

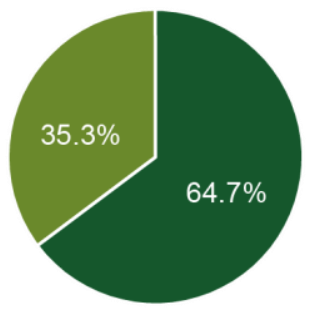

Single VT Dual VT
C

\section{VGLUT1}

VGLUT2

VGLUT3

VGLUT1-ZnT3

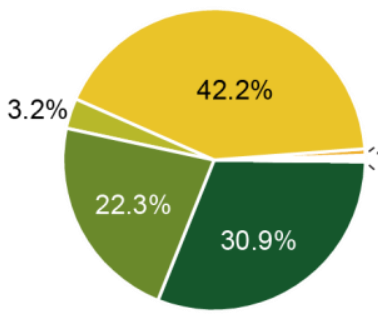

Glutamatergic SVs

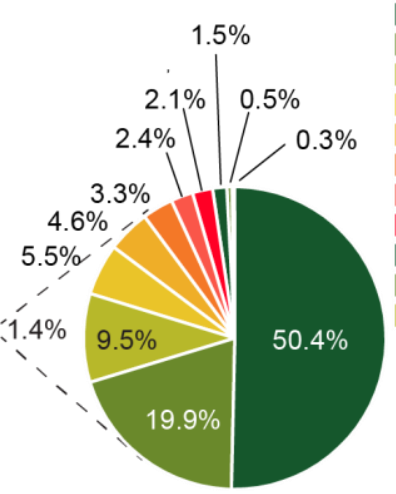

D GABAergic SVs

E GABAGlutamatergic SVs

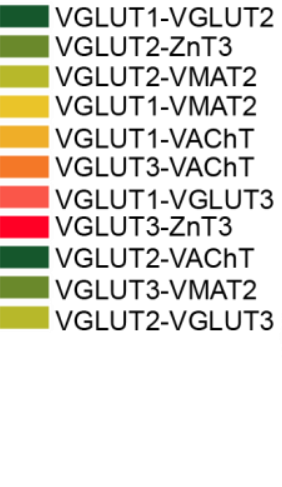

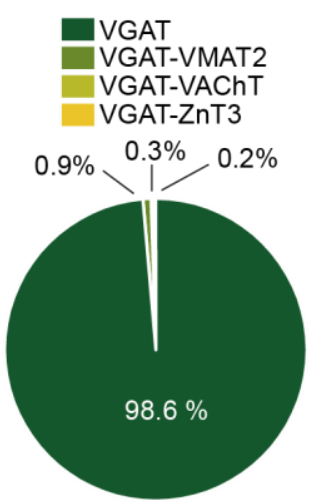
VGLUT3-VGAT

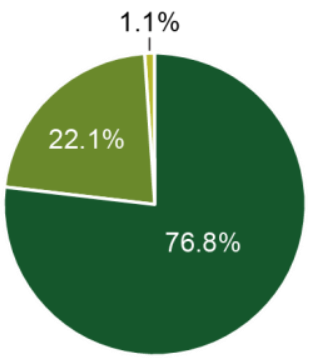

Figure 3. SVs are highly diverse based on the phenotype of expressed vesicular transporters(A) Bar graph quantifying the abundance of different vesicle classes containing either one (below dashed red line) or two distinct VTs (above the red dashed line). The numbers next to each bar denotes the actual value. Inset- representative electron immuno microscopy (IEM) image displaying SVs associated with gold nano-particles specific for VGLUT1 $(5 \mathrm{~nm})$ and ZnT3 $(10 \mathrm{~nm})$. (B) Pie diagram showing broad classification of SVs types -containing (1) only one of the three VGLUTs or (2) only VGAT or (3) VGLUTs coupled with another VT (except VGAT) or (4) VGAT coupled with another VT (except VGLUTs) or (5) one of the VGLUTs and VGAT. (C) Left, Pie diagram showing the composition of glutamatergic vesicles expressing either one of the three vesicular glutamatergic 
transporters (VGLUT1-3) or in association with another VT. The small components are visualized by an exploding pie chart recalibrated to $100 \%$ for greater visibility. (D) Similar pie chart, as shown in C, for GABAergic vesicles expressing VGAT. (E) Similar pie chart, as shown in C, for glutamatergic as well as GABAergic SVs expressing VGAT and one of the three VGLUTs. Bar graph values are means \pm SEM. Scale bar in $3 \mathrm{~A}$ inset $=50 \mathrm{~nm}$ 
A

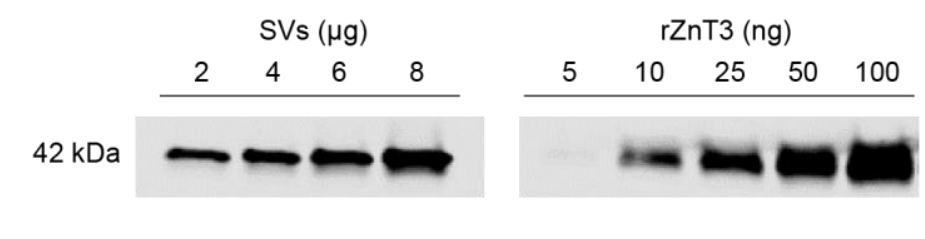

B
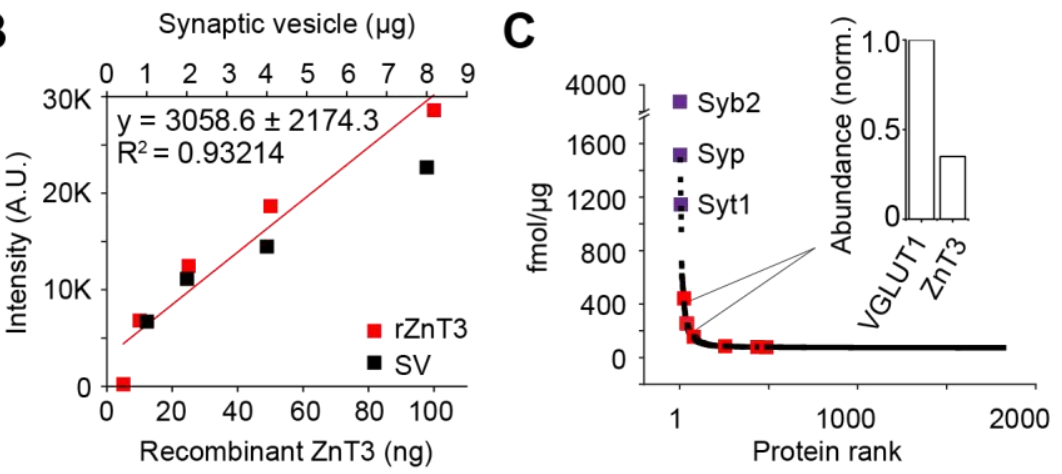

Figure 4. ZnT3 is a highly abundant SV protein specifically enriched in VGLUT1 positive vesicles (A) An example quantitative immunoblot for ZnT3 in SVs using purified recombinant ZnT3 protein as standard (rZnT3). Isolated SVs and rZnT3 were loaded onto the same gel at specified concentrations. (B) Scatter plot for quantification of ZnT3 abundance in SVs following quantitative immunoblotting using rZnT3 standard curve fitted with a linear function. (C) SV proteins ranked by their abundance as estimated using quantitative mass spectrometry (MS). Relative abundance of VGLUT1 and ZnT3 is shown as an inset. Selected proteins are highlighted for visibility; purple for specified SV markers and red for the VTs investigated in the current study. 

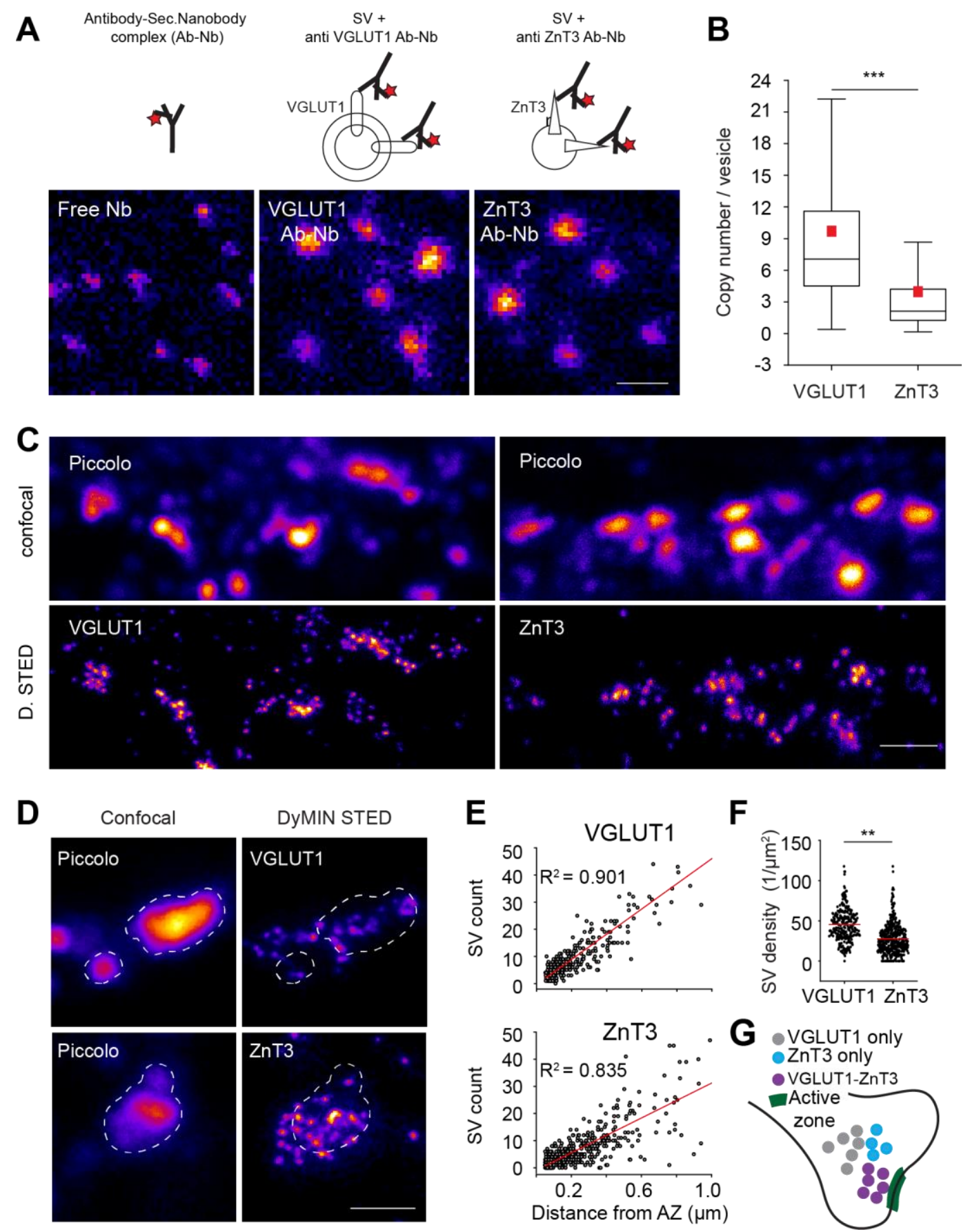

\section{Figure 5. Heterogeneous pools of SVs containing VGLUT1, ZnT3 and VGLUT1-ZnT3 reside at} individual synapses

(A) Top- illustration of stoichiometric labeling of VGLUT1 (middle) and ZnT3 (right) in isolated SVs using monoclonal primary antibodies and secondary nanobodies conjugated with fixed number of fluorophores The left illustration shows free primary antibody-secondary nanobody complex (Ab$\mathrm{Nb}$ ). Bottom- representative DyMIN STED images of free Ab-Nb (left) and of isolated SVs stoichiometrically labeled against VGLUT1 (middle) and ZnT3 (right). (B) Box plot quantifying the copy number of VGLUT1 and ZnT3 in individual SVs (n=3, p<0.00X, significant test). (C) Representative dendritic segments from hippocampal culture neurons showing VGLUT1 and ZnT3 
vesicles (DyMIN STED) in boutons stained with Piccolo (confocal), a presynaptic marker. (D) Magnified synaptic areas displaying Piccolo and super resolved VGLUT1 or ZnT3 positive SVs. (E) Scatter plot showing the correlation between SV count and the distance from active zone (AZ) for VGLUT1 (top) and ZnT3 (bottom) SVs at individual synapses. The data points were fitted with linear regression. (F) Bar plot comparing the density of VGLUT1 and ZnT3 positive SVs at individual synapses $(* * \mathrm{P}<0.05, \mathrm{n}=3,287$ VGLUT1 and 471 ZnT3 synapses, unpaired $t$-test). (G) Illustration depicting typical localization of VGLUT1-, ZnT3- and VGLUT1-ZnT3 positive SVs at a presynaptic compartment. Scale bars in $(A)=100 \mathrm{~nm} ;(C$ and $D)=1 \mu \mathrm{m}$. 


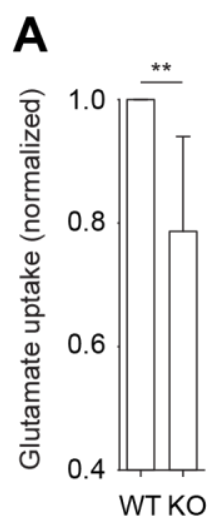

B
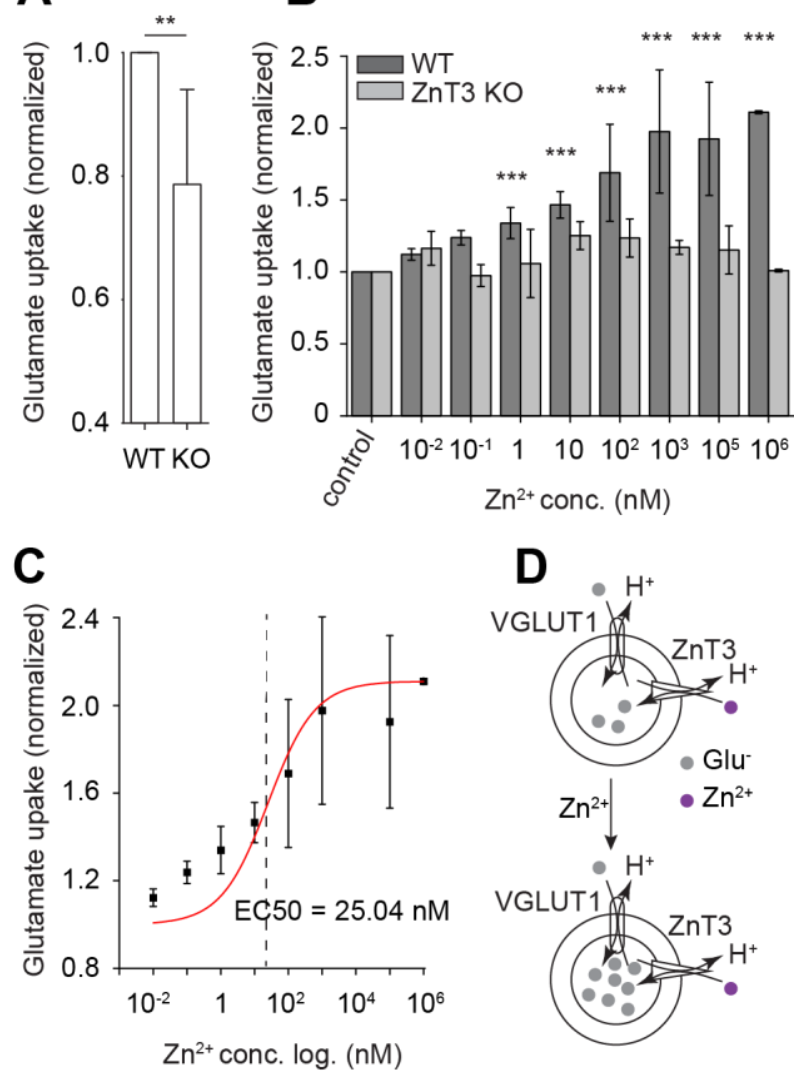

Figure 6. $\mathrm{Zn}^{2+}$ facilitates vesicular glutamate uptake by a mechanism mediated by ZnT3

(A) Bar graph showing enhanced basal vesicular glutamate uptake in isolated SVs (LP2 fraction) in $\mathrm{ZnT} 3$ wildtype mice (WT) in comparison to knockout (KO, ${ }^{*} \mathrm{P}<0.05, \mathrm{n}=6$ experiments, paired $t$-test). (B) Bar graph showing enhancement of glutamate uptake in increasing concentrations of $\mathrm{Zn}^{2+}$ in WT, which is absent in SVs prepared from ZnT3 KO mice $(* * * \mathrm{P}<0.001, \mathrm{n}=6$ experiments, two-way ANOVA). (C) Scatter plot showing dose-dependent facilitation of glutamate uptake by $\mathrm{Zn}^{2+}$. The data points were fitted with a sigmoidal function (Logistic5) to calculate the EC50 value. (D) Illustration of enhanced vesicular glutamate uptake by $\mathrm{Zn}^{2+}$. All values are means $\pm \mathrm{SEM}$. 
A

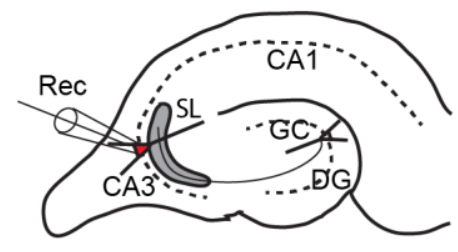

B

\section{ZnT3 WT}

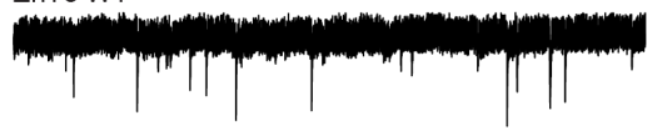

$\mathrm{ZnT3} \mathrm{WT}+\mathrm{Zn}^{2+}$

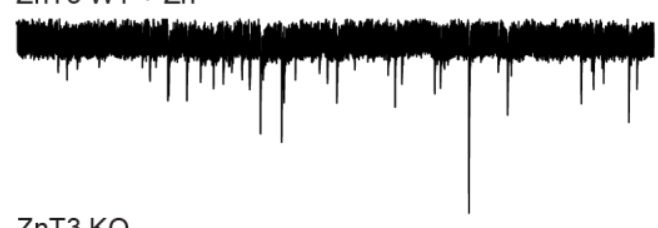

ZnT3 KO

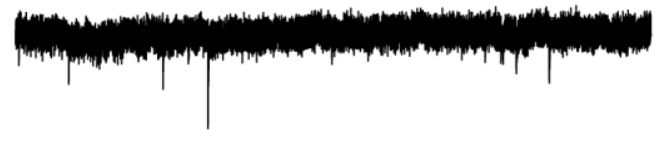

$\mathrm{ZnT3} \mathrm{KO}+\mathrm{Zn}^{2+}$

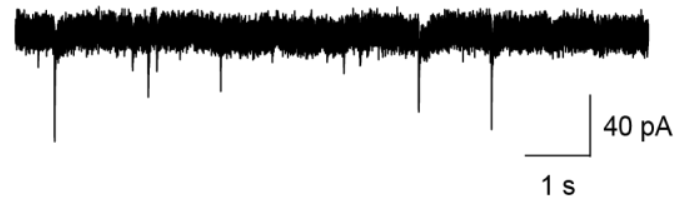

$\mathbf{E}$

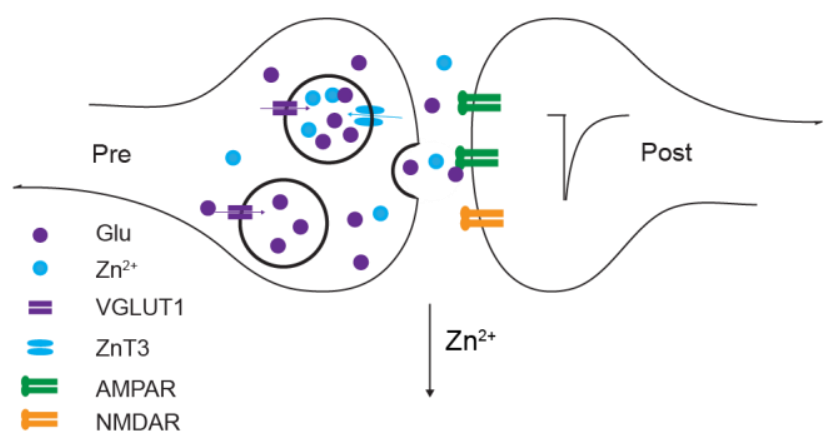

Wildtype

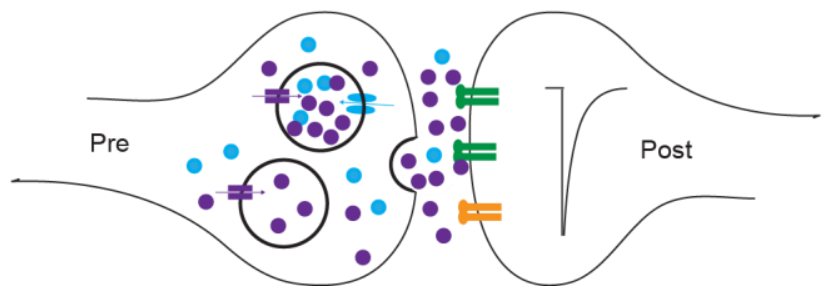

C
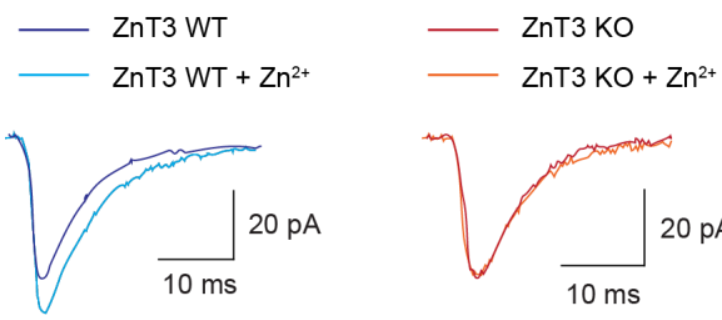

D
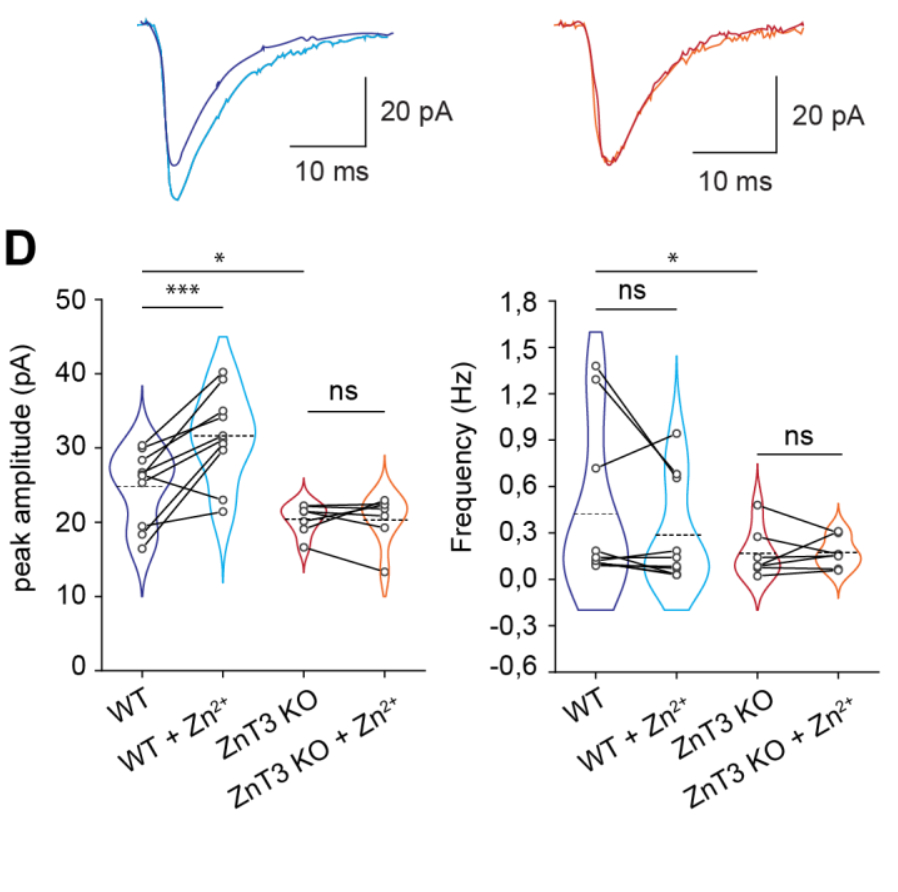

ZnT3 knockout
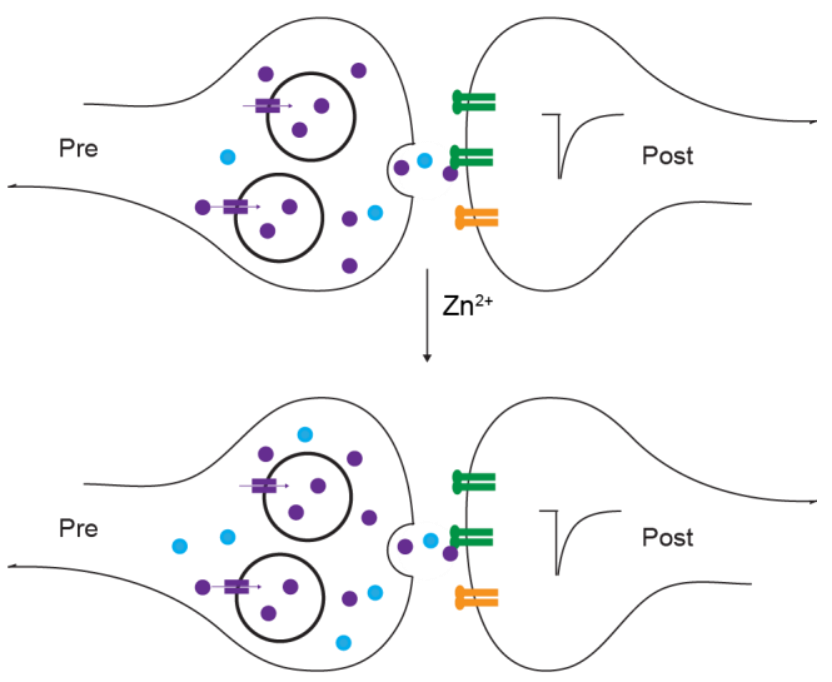

Figure 7. Ambient $\mathrm{Zn}^{2+}$ increases synaptic quantal size via $\mathrm{ZnT3}$

(A) Scheme of whole-cell recordings performed on hippocampal CA3 neurons. (B) Representative traces of mEPSC activity during baseline recording and after $\mathrm{Zn}^{2+}$ application in $\mathrm{ZnT3}$ wildtype (WT) and knockout (KO) mice. (C) Average traces showing time-course of mEPSCs during baseline and after $\mathrm{Zn}^{2+}$ application in WT (left, decay, $6.6 \pm 0.4 \mathrm{~ms}$ during baseline vs $7.48 \pm 0.7 \mathrm{~ms}$ after $\mathrm{Zn}^{2+}, \mathrm{p}>0.2$, rise time, $1.0 \pm 0.16 \mathrm{~ms}$ during baseline vs $1.1 \pm 01 \mathrm{~ms}$ after $\mathrm{Zn}^{2+}, \mathrm{p}>0.5$, paired t-test) and $\mathrm{KO}$ (right, 
decay: $7.8 \pm 1.2 \mathrm{~ms}$ during baseline vs $6.8 \pm 0.7 \mathrm{~ms}$ after $\mathrm{Zn}^{2+}, \mathrm{p}>0.20$ paired t-test; rise time: $1.15 \pm 0.14$ ms during baseline vs $1.1 \pm 0.1 \mathrm{~ms}$ after $\mathrm{Zn}^{2+}, \mathrm{p}>0.5$, paired t-test). (D) Violin plots comparing the peak amplitude (left) and frequency (right) of mEPSCs between baseline recordings and after $\mathrm{Zn}^{2+}$ application in ZnT3 WT and KO mice. Left: The baseline peak amplitude of mEPSCs was decreased in the KO compared to WT mice $\left(24.9 \pm 1.5 \mathrm{pA}\right.$ in WT, $\mathrm{n}=10 ; 20.73 \pm 0.7 \mathrm{pA}$ in $\mathrm{KO}, \mathrm{n}=7$; ${ }^{*} \mathrm{p}<0.05$, ttest). Following $\mathrm{Zn}^{2+}$ application, the peak amplitude significantly increased in the WT $(24.9 \pm 1.5 \mathrm{pA}$ during baseline; $31.2 \pm 1.9 \mathrm{pA}$ after $\mathrm{Zn}^{2+}$ application, $\mathrm{n}=10$, ***p<0.002, paired t-test) and not in the KO mice (20.7 $\pm 0.7 \mathrm{pA}$ during baseline; $20.4 \pm 1.2 \mathrm{pA}$ after $\mathrm{Zn}^{2+}$ application, $\mathrm{n}=7, \mathrm{p}>0.5$, paired t-test). Right: The baseline frequency was slightly reduced in the WT when compared to KO (WT, $0.42 \pm 0.16$ $\mathrm{Hz}, \mathrm{n}=10 ; \mathrm{KO}, 0.16 \pm 0.06 \mathrm{~Hz}, \mathrm{n}=7, \mathrm{p}<0.05$, t-test). Following $\mathrm{Zn}^{2+}$ application, the frequency did not change in both WT $\left(0.42 \pm 0.1 \mathrm{~Hz}\right.$ during baseline; $0.28 \pm 0.1$ after $\mathrm{Zn}^{2+}, \mathrm{p}>0.1$, paired t-test, $\left.\mathrm{n}=10\right)$ and KO (0.16 \pm 0.06 during baseline; $0.17 \pm 0.03$ after $\mathrm{Zn}^{2+}$ application, $\mathrm{n}=7, \mathrm{p}>0.5$, paired t-test). (E) Illustration showing the significance of colocalization of VGLUT1 and ZnT3 on same synaptic vesicles in the regulation of glutamate quantal size during synaptic activity. Top left: colocalization of VGLUT1 and ZnT3 leads to co-uptake and corelease of glutamate and $\mathrm{Zn}^{2+}$ from same SV pools in resting conditions. Bottom left: In the presence of ambient $\mathrm{Zn}^{2+}$, more glutamate is taken up into SVs expressing both VGLUT1 and ZnT3 leading to enhanced glutamate release and postsynaptic response. Top right: genetic deletion of ZnT3 leads to reduced vesicular glutamate content and postsynaptic response at resting conditions. Bottom right: Absence of ZnT3 does not affect vesicular glutamate content or postsynaptic response in the presence of ambient $\mathrm{Zn}^{2+}$ demonstrating that localization of the protein in VGLUT1 SVs is critical for $\mathrm{Zn}^{2+}$ mediated regulation of glutamatergic neurotransmission. These data emphasize coupling of transporter activity of VGLUT1 and ZnT3 leading to regulation of synaptic quantal size. The regulation of substrate uptake by $\mathrm{Zn}^{2+}$ in synaptic vesicles assumes significance because of striking similarity to the well-known $\mathrm{Zn}^{2+}$ mediated uptake of insulin in secretory vesicles of pancreatic beta cells via ZnT8. 


\section{REFERENCES}

Anderson, C.T., Kumar, M., Xiong, S., and Tzounopoulos, T. (2017). Cell-specific gain modulation by synaptically released zinc in cortical circuits of audition. Elife 6 .

Bellocchio, E.E., Reimer, R.J., Fremeau, R.T., Jr., and Edwards, R.H. (2000). Uptake of glutamate into synaptic vesicles by an inorganic phosphate transporter. Science 289, 957-960.

Brown, C.E., and Dyck, R.H. (2002). Rapid, experience-dependent changes in levels of synaptic zinc in primary somatosensory cortex of the adult mouse. J Neurosci 22, 2617-2625.

Dyck, R.H., Chaudhuri, A., and Cynader, M.S. (2003). Experience-dependent regulation of the zincergic innervation of visual cortex in adult monkeys. Cereb Cortex 13, 1094-1109.

Edwards, R.H. (2007). The neurotransmitter cycle and quantal size. Neuron 55, 835-858.

Eide, D.J. (2006). Zinc transporters and the cellular trafficking of zinc. Biochim Biophys Acta 1763, 711-722.

El Mestikawy, S., Wallen-Mackenzie, A., Fortin, G.M., Descarries, L., and Trudeau, L.E. (2011). From glutamate co-release to vesicular synergy: vesicular glutamate transporters. Nat Rev Neurosci 12, 204-216.

Eriksen, J., Chang, R., McGregor, M., Silm, K., Suzuki, T., and Edwards, R.H. (2016). Protons Regulate Vesicular Glutamate Transporters through an Allosteric Mechanism. Neuron 90, 768-780. Farsi, Z., Preobraschenski, J., van den Bogaart, G., Riedel, D., Jahn, R., and Woehler, A. (2016). Single-vesicle imaging reveals different transport mechanisms between glutamatergic and GABAergic vesicles. Science 351, 981-984.

Fasano, C., Rocchetti, J., Pietrajtis, K., Zander, J.F., Manseau, F., Sakae, D.Y., Marcus-Sells, M., Ramet, L., Morel, L.J., Carrel, D., et al. (2017). Regulation of the Hippocampal Network by VGLUT3-Positive CCK- GABAergic Basket Cells. Front Cell Neurosci 11, 140.

Frahm, S., Antolin-Fontes, B., Gorlich, A., Zander, J.F., Ahnert-Hilger, G., and Ibanez-Tallon, I. (2015). An essential role of acetylcholine-glutamate synergy at habenular synapses in nicotine dependence. Elife 4, e11396.

Frederickson, C.J., Koh, J.Y., and Bush, A.I. (2005). The neurobiology of zinc in health and disease. Nat Rev Neurosci 6, 449-462.

Geumann, U., Barysch, S.V., Hoopmann, P., Jahn, R., and Rizzoli, S.O. (2008). SNARE function is not involved in early endosome docking. Mol Biol Cell 19, 5327-5337.

Goch, W., and Bal, W. (2020). Stochastic or Not? Method To Predict and Quantify the Stochastic Effects on the Association Reaction Equilibria in Nanoscopic Systems. J Phys Chem A 124, 14211428.

Goh, G.Y., Huang, H., Ullman, J., Borre, L., Hnasko, T.S., Trussell, L.O., and Edwards, R.H. (2011). Presynaptic regulation of quantal size: $\mathrm{K}+\mathrm{H}+$ exchange stimulates vesicular glutamate transport. Nat Neurosci 14, 1285-1292.

Gottfert, F., Pleiner, T., Heine, J., Westphal, V., Gorlich, D., Sahl, S.J., and Hell, S.W. (2017). Strong signal increase in STED fluorescence microscopy by imaging regions of subdiffraction extent. Proc Natl Acad Sci U S A 114, 2125-2130.

Granger, A.J., Wallace, M.L., and Sabatini, B.L. (2017). Multi-transmitter neurons in the mammalian central nervous system. Curr Opin Neurobiol 45, 85-91.

Gras, C., Amilhon, B., Lepicard, E.M., Poirel, O., Vinatier, J., Herbin, M., Dumas, S., Tzavara, E.T., Wade, M.R., Nomikos, G.G., et al. (2008). The vesicular glutamate transporter VGLUT3 synergizes striatal acetylcholine tone. Nat Neurosci 11, 292-300.

Gronborg, M., Pavlos, N.J., Brunk, I., Chua, J.J., Munster-Wandowski, A., Riedel, D., AhnertHilger, G., Urlaub, H., and Jahn, R. (2010). Quantitative comparison of glutamatergic and 
GABAergic synaptic vesicles unveils selectivity for few proteins including MAL2, a novel synaptic vesicle protein. J Neurosci 30, 2-12.

Heine, J., Reuss, M., Harke, B., D'Este, E., Sahl, S.J., and Hell, S.W. (2017). Adaptive-illumination STED nanoscopy. Proc Natl Acad Sci U S A 114, 9797-9802.

Herzog, E., Takamori, S., Jahn, R., Brose, N., and Wojcik, S.M. (2006). Synaptic and vesicular colocalization of the glutamate transporters VGLUT1 and VGLUT2 in the mouse hippocampus. J Neurochem 99, 1011-1018.

Higley, M.J., Gittis, A.H., Oldenburg, I.A., Balthasar, N., Seal, R.P., Edwards, R.H., Lowell, B.B., Kreitzer, A.C., and Sabatini, B.L. (2011). Cholinergic interneurons mediate fast VGluT3-dependent glutamatergic transmission in the striatum. PLoS One 6, e19155.

Hnasko, T.S., Chuhma, N., Zhang, H., Goh, G.Y., Sulzer, D., Palmiter, R.D., Rayport, S., and Edwards, R.H. (2010). Vesicular glutamate transport promotes dopamine storage and glutamate corelease in vivo. Neuron 65, 643-656.

Hnasko, T.S., and Edwards, R.H. (2012). Neurotransmitter corelease: mechanism and physiological role. Annu Rev Physiol 74, 225-243.

Juge, N., Gray, J.A., Omote, H., Miyaji, T., Inoue, T., Hara, C., Uneyama, H., Edwards, R.H., Nicoll, R.A., and Moriyama, Y. (2010). Metabolic control of vesicular glutamate transport and release. Neuron 68, 99-112.

Juge, N., Yoshida, Y., Yatsushiro, S., Omote, H., and Moriyama, Y. (2006). Vesicular glutamate transporter contains two independent transport machineries. J Biol Chem 281, 39499-39506.

Kalappa, B.I., Anderson, C.T., Goldberg, J.M., Lippard, S.J., and Tzounopoulos, T. (2015). AMPA receptor inhibition by synaptically released zinc. Proc Natl Acad Sci U S A 112, 15749-15754. Kawahara, M., Kato-Negishi, M., and Tanaka, K.I. (2020). Amyloids: Regulators of Metal Homeostasis in the Synapse. Molecules 25.

Krezel, A., and Maret, W. (2016). The biological inorganic chemistry of zinc ions. Arch Biochem Biophys 611, 3-19.

Lavoie, N., Jeyaraju, D.V., Peralta, M.R., 3rd, Seress, L., Pellegrini, L., and Toth, K. (2011).

Vesicular zinc regulates the $\mathrm{Ca} 2+$ sensitivity of a subpopulation of presynaptic vesicles at hippocampal mossy fiber terminals. J Neurosci 31, 18251-18265.

Li, F., Eriksen, J., Finer-Moore, J., Chang, R., Nguyen, P., Bowen, A., Myasnikov, A., Yu, Z., Bulkley, D., Cheng, Y., et al. (2020). Ion transport and regulation in a synaptic vesicle glutamate transporter. Science 368, 893-897.

Liu, S., Plachez, C., Shao, Z., Puche, A., and Shipley, M.T. (2013). Olfactory bulb short axon cell release of GABA and dopamine produces a temporally biphasic inhibition-excitation response in external tufted cells. J Neurosci 33, 2916-2926.

Malkusch, S., Endesfelder, U., Mondry, J., Gelleri, M., Verveer, P.J., and Heilemann, M. (2012).

Coordinate-based colocalization analysis of single-molecule localization microscopy data.

Histochem Cell Biol 137, 1-10.

Marvin, J.S., Scholl, B., Wilson, D.E., Podgorski, K., Kazemipour, A., Muller, J.A., Schoch, S., Quiroz, F.J.U., Rebola, N., Bao, H., et al. (2018). Stability, affinity, and chromatic variants of the glutamate sensor iGluSnFR. Nat Methods 15, 936-939.

McAllister, B.B., and Dyck, R.H. (2017). Zinc transporter 3 (ZnT3) and vesicular zinc in central nervous system function. Neurosci Biobehav Rev 80, 329-350.

Minckley, T.F., Zhang, C., Fudge, D.H., Dischler, A.M., LeJeune, K.D., Xu, H., and Qin, Y. (2019). Sub-nanomolar sensitive GZnP3 reveals TRPML1-mediated neuronal $\mathrm{Zn}(2+)$ signals. Nat Commun 10, 4806.

Morales, M., and Margolis, E.B. (2017). Ventral tegmental area: cellular heterogeneity, connectivity and behaviour. Nat Rev Neurosci 18, 73-85. 
Olsen, S.R., Bortone, D.S., Adesnik, H., and Scanziani, M. (2012). Gain control by layer six in cortical circuits of vision. Nature 483, 47-52.

Palmiter, R.D., Cole, T.B., Quaife, C.J., and Findley, S.D. (1996). ZnT-3, a putative transporter of zinc into synaptic vesicles. Proc Natl Acad Sci U S A 93, 14934-14939.

Pociot, F., and Lernmark, A. (2016). Genetic risk factors for type 1 diabetes. Lancet 387, 2331-2339. Pothos, E.N., Larsen, K.E., Krantz, D.E., Liu, Y., Haycock, J.W., Setlik, W., Gershon, M.D., Edwards, R.H., and Sulzer, D. (2000). Synaptic vesicle transporter expression regulates vesicle phenotype and quantal size. J Neurosci 20, 7297-7306.

Preobraschenski, J., Cheret, C., Ganzella, M., Zander, J.F., Richter, K., Schenck, S., Jahn, R., and Ahnert-Hilger, G. (2018). Dual and Direction-Selective Mechanisms of Phosphate Transport by the Vesicular Glutamate Transporter. Cell Rep 23, 535-545.

Preobraschenski, J., Zander, J.F., Suzuki, T., Ahnert-Hilger, G., and Jahn, R. (2014). Vesicular glutamate transporters use flexible anion and cation binding sites for efficient accumulation of neurotransmitter. Neuron 84, 1287-1301.

Ren, J., Qin, C., Hu, F., Tan, J., Qiu, L., Zhao, S., Feng, G., and Luo, M. (2011). Habenula "cholinergic" neurons co-release glutamate and acetylcholine and activate postsynaptic neurons via distinct transmission modes. Neuron 69, 445-452.

Romero-Hernandez, A., Simorowski, N., Karakas, E., and Furukawa, H. (2016). Molecular Basis for Subtype Specificity and High-Affinity Zinc Inhibition in the GluN1-GluN2A NMDA Receptor Amino-Terminal Domain. Neuron 92, 1324-1336.

Root, D.H., Mejias-Aponte, C.A., Zhang, S., Wang, H.L., Hoffman, A.F., Lupica, C.R., and Morales, M. (2014). Single rodent mesohabenular axons release glutamate and GABA. Nat Neurosci 17, 1543-1551.

Root, D.H., Zhang, S., Barker, D.J., Miranda-Barrientos, J., Liu, B., Wang, H.L., and Morales, M. (2018). Selective Brain Distribution and Distinctive Synaptic Architecture of Dual GlutamatergicGABAergic Neurons. Cell Rep 23, 3465-3479.

Salazar, G., Craige, B., Love, R., Kalman, D., and Faundez, V. (2005). Vglut1 and ZnT3 co-targeting mechanisms regulate vesicular zinc stores in PC12 cells. J Cell Sci 118, 1911-1921.

Sanford, L., Carpenter, M.C., and Palmer, A.E. (2019). Intracellular $\mathrm{Zn}(2+)$ transients modulate global gene expression in dissociated rat hippocampal neurons. Sci Rep 9, 9411.

Saunders, A., Granger, A.J., and Sabatini, B.L. (2015). Corelease of acetylcholine and GABA from cholinergic forebrain neurons. Elife 4.

Schenck, S., Wojcik, S.M., Brose, N., and Takamori, S. (2009). A chloride conductance in VGLUT1 underlies maximal glutamate loading into synaptic vesicles. Nat Neurosci 12, 156-162.

Sengupta, A., Bocchio, M., Bannerman, D.M., Sharp, T., and Capogna, M. (2017). Control of Amygdala Circuits by 5-HT Neurons via 5-HT and Glutamate Cotransmission. J Neurosci 37, 17851796.

Shabel, S.J., Proulx, C.D., Piriz, J., and Malinow, R. (2014). Mood regulation. GABA/glutamate corelease controls habenula output and is modified by antidepressant treatment. Science 345, 14941498.

Sograte-Idrissi, S., Schlichthaerle, T., Duque-Afonso, C.J., Alevra, M., Strauss, S., Moser, T., Jungmann, R., Rizzoli, S.O., and Opazo, F. (2020). Circumvention of common labelling artefacts using secondary nanobodies. Nanoscale 12, 10226-10239.

Takacs, V.T., Cserep, C., Schlingloff, D., Posfai, B., Szonyi, A., Sos, K.E., Kornyei, Z., Denes, A., Gulyas, A.I., Freund, T.F., et al. (2018). Co-transmission of acetylcholine and GABA regulates hippocampal states. Nat Commun 9, 2848. 
Takamori, S., Holt, M., Stenius, K., Lemke, E.A., Gronborg, M., Riedel, D., Urlaub, H., Schenck, S., Brugger, B., Ringler, P., et al. (2006). Molecular anatomy of a trafficking organelle. Cell 127, 831846.

Takamori, S., Rhee, J.S., Rosenmund, C., and Jahn, R. (2001). Identification of differentiationassociated brain-specific phosphate transporter as a second vesicular glutamate transporter (VGLUT2). J Neurosci 21, RC182.

Takamori, S., Riedel, D., and Jahn, R. (2000). Immunoisolation of GABA-specific synaptic vesicles defines a functionally distinct subset of synaptic vesicles. J Neurosci 20, 4904-4911.

Taoufiq, Z., Ninov, M., Villar-Briones, A., Wang, H.Y., Sasaki, T., Roy, M.C., Beauchain, F., Mori, Y., Yoshida, T., Takamori, S., et al. (2020). Hidden proteome of synaptic vesicles in the mammalian brain. Proc Natl Acad Sci U S A 117, 33586-33596.

Trist, B.G., Hare, D.J., and Double, K.L. (2018). A Proposed Mechanism for Neurodegeneration in Movement Disorders Characterized by Metal Dyshomeostasis and Oxidative Stress. Cell Chem Biol 25, 807-816.

Tritsch, N.X., Granger, A.J., and Sabatini, B.L. (2016). Mechanisms and functions of GABA corelease. Nat Rev Neurosci 17, 139-145.

Trudeau, L.E., and El Mestikawy, S. (2018). Glutamate Cotransmission in Cholinergic, GABAergic and Monoamine Systems: Contrasts and Commonalities. Front Neural Circuits 12, 113.

Vaaga, C.E., Borisovska, M., and Westbrook, G.L. (2014). Dual-transmitter neurons: functional implications of co-release and co-transmission. Curr Opin Neurobiol 29, 25-32.

Varga, V., Losonczy, A., Zemelman, B.V., Borhegyi, Z., Nyiri, G., Domonkos, A., Hangya, B., Holderith, N., Magee, J.C., and Freund, T.F. (2009). Fast synaptic subcortical control of hippocampal circuits. Science 326, 449-453.

Vergnano, A.M., Rebola, N., Savtchenko, L.P., Pinheiro, P.S., Casado, M., Kieffer, B.L., Rusakov, D.A., Mulle, C., and Paoletti, P. (2014). Zinc dynamics and action at excitatory synapses. Neuron 82, 1101-1114.

Walker, M.C., Ruiz, A., and Kullmann, D.M. (2001). Monosynaptic GABAergic signaling from dentate to CA3 with a pharmacological and physiological profile typical of mossy fiber synapses. Neuron 29, 703-715.

Wilhelm, B.G., Mandad, S., Truckenbrodt, S., Krohnert, K., Schafer, C., Rammner, B., Koo, S.J., Classen, G.A., Krauss, M., Haucke, V., et al. (2014). Composition of isolated synaptic boutons reveals the amounts of vesicle trafficking proteins. Science 344, 1023-1028.

Xue, J., Xie, T., Zeng, W., Jiang, Y., and Bai, X.C. (2020). Cryo-EM structures of human ZnT8 in both outward- and inward-facing conformations. Elife 9.

Zeisel, A., Hochgerner, H., Lonnerberg, P., Johnsson, A., Memic, F., van der Zwan, J., Haring, M., Braun, E., Borm, L.E., La Manno, G., et al. (2018). Molecular Architecture of the Mouse Nervous System. Cell 174, 999-1014 e1022.

Zimmermann, J., Herman, M.A., and Rosenmund, C. (2015). Co-release of glutamate and GABA from single vesicles in GABAergic neurons exogenously expressing VGLUT3. Front Synaptic Neurosci 7, 16. 